\title{
Ueber den Begriff der Irreductibilität in der Theorie der linearen Differentialgleichungen.
}

(Von Herrn G. Frobenius.)

Das allgemeine Integral einer gewöhnlichen Differentialgleichung $\lambda_{\text {ter }}$ Ordnung hängt nicht von einer, sondern von $\lambda+1$ unabhängigen Veränderlichen $a b$, von denen die eine, nach welcher die gesuchte Function in der gegebenen Gleichung differentiirt wird, die Variable genannt -wird, die übrigen $\lambda$ aber, welche nicht in die Differentialgleichung eingehen, als willkürliche Constanten bezeichnet werden. In Folge davon ist man gewohnt, eine Differentialgleichung als das Resultat der Elimination der willkürlichen Constanten aus einer Function und ihren Ableitungen aufzufassen.

In der neueren Zeit aber hat sich noch eine andere, von der früheren etwas abweichende Anschauung über die Differentialgleichungen geltend gemacht. Man bemerkte nämlich, dass, wenn ein so unbestimmter Ausdruck erlaubt ist, die Integrale der Differentialgleichungen meistens in höherem Maasse vieldeutig sind, als ihre Coefficienten, dass also, um bei dem einfachsten Falle stehen zu bleiben, die Integrale von Differentialgleichungen mit eindeutigen Coefficienten in der Regel mehrwerthige Functionen sind: Daher ist es jetzt auch gestattet, die Differentialgleichungen als Gleichungen aufzufassen, in deren Coefficienten die Vieldeutigkeit der sie befriedigenden Functionen ganz oder zum Theil verwischt erscheint, und die deshalb zur Definition mehrwerthiger Functionen in besonderer Weise geeignet sind.

Nach der ersten Anschaungsweise heisst eine Differentialgleichung $\lambda$ ter Ordnung vollständig integriren: einen ihr genügenden Ausdruck angeben, der $\lambda$ von einander unabhängige willkürliche Constanten enthält; nach der zweiten: den Verlauf der sie befriedigenden analytischen Functionen durch die ganze Ebene, insbesondere in der Umgebung der Verzweigungs - und Unstetigkeitswerthe verfolgen.' Die erste Aufgabe hat für die angewandte, die zweite für die reine Analysis höheres Interesse. Indessen ist nicht zu verkennen, dass der Unterschied zwischen beiden Betrachtungsweisen nicht so gross ist, wie es vielleicht den Anschein hat, und mehr die Form als die Sache angeht. 
Meine Bemühungen, den Begriff der Irreductibilität, welcher für die ganze Mathematik und namentlich für die Theorie der algebraischen Gleichungen von einer so hervorragenden Wichligkeit ist, auch in die Lehre von den Differentialgleichungen einzuführen, blieben so lange erfolglos, als ich die frühere Anschaungsweise festhielt. Den Grund davon wird eine Vergleichung mit den algebraischen Gleichungen leicht aufhellen. So lange man deren linke Seite als ein Product von linearen Factoren auffasst, sind sie stets reductibel. Geht man dagegen von einer auf irgend eine Weise, z. B. durch einen periodischen Kettenbruch gegebenen irrationalen Zahl aus und fragt nach den -algebraischen Gleichungen mit rationalen Coefficienten, denen sie genügt, so wird man sofort darauf geführt, die eine, welche in allen andern als Factor auftritt, als irreductibel, die übrigen aber als reductibel zu bezeichnen.

Von der zweiten Anschauung über die Differentialgleichungen ausgehend, nenne ich eine Differentialgleichung, deren Coefficienten in einem gewissen Theile der Ebene eindeutig definirte analytische Functionen sind, irreductibel, wenn sie mit keiner Differentialgleichung niedrigerer Ordnung oder bei gleicher Ordnung niedrigeren Grades, deren Coefficienten in demselben Flächenstück einwerthige analytische Functionen sind, ein Integral gemeinsam hat. Dieser Definition gemäss ist z. B. jede algebraische Differentialgleichung, unter deren Integralen sich algebraische Functionen befinden, reductibel, weil sie mit einer algebraischen Differentialgleichung nullter Ordnung Integrale gemeinsam hat.

Weil aber von der allgemeinen Theorie der Differentialgleichungen wenig mehr, als der Salz des Herrn Weierstrass über die Existenz ihrer Integrale bekannt ist, so werde ich mich, um den Begriff der Irreductibilität zu erläutern, auf die Differentialgleichungen von einer höheren als der ersten Ordnung beschränken, deren Theorie allein genauer erforscht ist, auf die linearen. Und da es mir bei der Einführung eines neuen Begriffs weniger auf Allgemeinheit, als auf Genauigkeit ankommt, so werde ich - nur die zuerst von Herrn Fuchs (dieses Journal Bd. 66, S. 146) untersuchte merkwürdige Klasse von homogenen linearen Differentialgleichungen in den Kreis meiner Untersuchungen ziehen, deren Integrale nur eine endliche Anzahl singulärer Stellen haben und an jeder derselben, $a$, mit einer endlichen Potenz von $x-a$ (oder $\frac{1}{x}$ für $a=\infty$ ) multiplicirt, endlich bleiben. Wenn eine solche mit einer anderen von derselben Beschaffenheit aber niedrigerer Ordnung ein In- 
tegral gemeinsam hat, so soll sie reductibel, im entgegengesetzten Falle irreductibel genannt werden. Wo im Folgenden von einer linearen Differentialgleichung schlechthin gesprochen wird, soll stets eine solche darunter verstanden werden. Ehe ich aber an die Herleitung der Eigenschaften der irreductibeln linearen Differentialgleichungen gehen kann, muss ich zwei Hülfssätze beweisen, auf denen die weitere Entwicklung zum grössten Theile beruht.

S. 1 .

Die Functionen $y_{1}, y_{2}, \ldots y_{\lambda}$ seien durch $\lambda$ nach ganzen positiven Potenzen von $x-x_{0}$ fortschreitende Reihen definirt, die innerhalb eines mitdem Radius $r$ um den Punkt $x_{0}$ beschriebenen Kreises sämmtlich convergiren. Wir beschränken alsdann die Veränderlichkeit von $x$ vorläufig auf die Fläche dieses Kreises. Besteht zwischen diesen Functionen eine homogene lineare Gleichung mit constanten Coefficienten

$$
c_{1} y_{1}+c_{2} y_{2}+\cdots+c_{\lambda} y_{\lambda}=0
$$

so ergiebt sich, indem man dieselbe wiederholt differentiirt, und aus ihr und den $\lambda-1$ ersten aus ihr abgeleiteten Gleichungen die Constanten $c_{1}, c_{2}, \ldots c_{i}$ eliminirt, das Verschwinden der Determinante

$$
\boldsymbol{D}=\Sigma \pm y_{1} y_{2}^{(1)} \ldots y_{\lambda}^{(\lambda-1)},
$$

in $\operatorname{der} y_{\alpha}^{(\beta)}$ die $\beta^{\text {te }}$ Ableitung von $y_{\alpha}$, hedeutet. Aber auch umgekehrt folgt aus dem.Verschwinden des Ausdrucks $D$, den wir die Determinante der Functionen $y_{1}, y_{2}, \ldots y_{\lambda}$ nennen wollen, das Bestehen einer homogenen linearen Gleichung mit constanten Coefficienten zwischen den $\lambda$ Functionen.

Um dies zu beweisen, nehmen wir zunächst an, dass die Unterdeterminante $(\lambda-1)^{\text {ten }}$ Grades, $D^{\prime}$, welche in der Entwicklung von $D$ den Coefficienten von $y_{\lambda}^{(\lambda-1)}$ bildet, nicht identisch verschwindet. Löst man dann die $\lambda-1$ homogenen linearen Gleichungen

$$
\left\{\begin{array}{c}
z_{1} y_{1}+z_{2} y_{2}+\cdots+z_{\lambda} y_{\lambda}=0 \\
z_{1} y_{1}^{(1)}+z_{2} y_{2}^{(1)}+\cdots+z_{\lambda} y_{\lambda}^{(1)}=0 \\
\cdot \cdots \\
z_{1} y_{1}^{(\lambda-2)}+z_{2} y_{2}^{(\lambda-2)}+\cdots+z_{\lambda} y_{\lambda}^{(\lambda-2)}=0
\end{array}\right.
$$

nach den Unbekannten $z_{1}, z_{2}, \ldots z_{l}$ auf, so erhält man gemäss unserer Vor- . aussetzung für die Verhältnisse

$$
\frac{z_{1}}{z_{l}}, \frac{z_{2}}{z_{\lambda}}, \cdots \frac{z_{\lambda-1}}{z_{\lambda}}
$$


völlig bestimmte endliche Functionen. Diese müssen in Folge der Annahme $D=0$ auch die Gleichung

$$
z_{1} y_{1}^{(\lambda-1)}+z_{2} y_{2}^{(\lambda-1)}+\cdots+z_{\lambda} y_{\lambda}^{(\lambda-1)}=0
$$

befriedigen. Mit Berücksichtigung derselben erhält man aus dem Gleichungssystem (A.) durch Differentiation das folgende:

$$
\left\{\begin{array}{c}
z_{1}^{\prime} y_{1}+z_{2}^{\prime} y_{2}+\cdots+z_{\lambda}^{\prime} y_{\lambda}=0, \\
z_{1}^{\prime} y_{1}^{(1)}+z_{2}^{\prime} y_{2}^{(1)}+\cdots+z_{\lambda}^{\prime} y_{\lambda}^{(1)}=0, \\
\cdot \cdots \\
z_{1}^{\prime} y_{1}^{(\lambda-2)}+z_{2}^{\prime} y_{2}^{(\lambda-2)}+\cdots+z_{\lambda}^{\prime} y_{\lambda}^{(\lambda-2)}=0,
\end{array}\right.
$$

in welchem $z_{x}^{\prime}$ die Ableitung von $z_{x}$ bezeichnet. Da aber durch die Gleichungen (A.), die Verhältnisse

$$
\frac{z_{1}}{z_{\lambda}}, \frac{z_{2}}{z_{\lambda}}, \ldots \frac{z_{\lambda-1}}{z_{\lambda}}
$$

vollständig bestimmt werden, so müssen sich aus dem mit (A.) identischen Gleichungssystem (B.) für die-Verhältnisse

$$
\frac{z_{1}^{\prime}}{z_{\lambda}^{\prime}}, \frac{z_{2}^{\prime}}{z_{\lambda}^{\prime}}, \ldots \frac{z_{\lambda-1}^{\prime}}{z_{\lambda}^{\prime}}
$$

dieselben Werthe ergeben, oder es muss für $\varkappa=1,2, \ldots \lambda-1$

$$
\frac{z_{x}}{z_{\lambda}}=\frac{z_{\alpha}^{\prime}}{z_{\lambda}^{\prime}}, \quad \frac{d}{d x} \frac{z_{x}}{z_{\lambda}}=0, \quad \frac{z_{x}}{z_{\lambda}}=\frac{c_{\varkappa}}{c_{\lambda}}
$$

sein, wenn $c_{1}, c_{2}, \ldots c_{\lambda}$ Constanten sind, von denen $c_{\lambda}$ willkürlich, aber von Null verschieden ist. Aus der ersten Gleichung des Systems (A.) folgt daher die Relation

$$
\text { (C.) } \quad c_{1} y_{1}+c_{2} y_{2}+\cdots+c_{\lambda} y_{\lambda}=0 \text {. }
$$

Wenn $D^{\prime}=0$ ist, aber die Unterdeterminante $(\lambda-2)^{\text {ten }}$ Grades $D^{\prime \prime}$, welche in $D^{\prime}$ den Coefficienten von $y_{\lambda-1}^{(\lambda-2)}$ bildet, nicht identisch verschwindet, so ergiebt sich auf demselben Wege eine Relation von der Form

$$
c_{1} y_{1}+c_{2} y_{2}+\cdots+c_{\lambda-1} y_{\lambda-1}=0
$$

in welcher $c_{\lambda-1}$ von Null verschieden ist. Indem man so weiter schliesst, beweist man, dass aus der Annahme $D=0$ stets eine Gleichung von der Form (C.) folgt, in welcher die Constanten $c_{1}, c_{2}, \ldots c_{k}$ nicht sämmtlich verschwinden, wenn man bedenkt, dass auch die Gleichung $y_{1}=0$ unter dieser Form enthalten ist.

Im Folgenden sollen mehrere Functionen von einander unabhängig ge- 
nannt werden, wenn żwischen ihnen keine homogene lineare Gleichung mit constanten Coefficienten besteht. Alsdaun können wir den Satz aussprechen:

Wenn mehrére Functionen von einander unabhängig sind, so ist ihre Determinante von Null verschieden; wenn sie aber nicht von einander unabhängig sind, so ist ihre Determinante gleich Null.

Derselbe ist zunächst nur für das Innere des mit dem Radius $r$ um den Punkt $x_{0}$ beschriebenen Kreises bewiesen. Nach einem bekannten Theorem der Functionentheorie ergiebt sich daraus seine Gültigkeit für alle Theile der Ebene, nach denen hin man die $\lambda$ Functionen sämmtlich fortsetzen kann, vorausgesetzt, dass man unter $y_{1}, y_{2}, \ldots y_{\lambda}$ stets simultane Werthe dieser Functionen versteht, d. h. solche, welche sie annehmen, wenn sie alle auf demselben Wege fortgesetzt werden.

Man lë̈tet den bewiesenen Satz gewöhnlich aus der Theorie der linearen Differentialgleichungen ab. Es kam mir hier darauf an, ihn unabhăngig von dieser Lehre zu begründen.

\section{\$. 2 .}

Seien wieder eine oder mehrere Functionen $u, v, w, \ldots$ durch Reihen definirt, die nach ganzen positiven Potenzen von $x-x_{0}$ fortschreiten und in der Umgebung des Punktes $x_{0}$ convergiren. Wir nehmen an, dass dieselben sich über die ganze Ebene mit Ausschluss einer endlichen Anzahl singulärer Stellen fortsetzen lassen und an jedem singulären Punkte, $a$, mit einer endlichen Potenz von $x-a$ (oder $\frac{1}{x}$ für $a=\infty$ ) multiplicirt, endlich bleiben. Denkt man sich, dass die unabhängige Veränderliche $x$ von $x_{0}$ aus alle möglichen durch keinen singulären Punkt hindurchführenden geschlossenen Wege durchläuft, so gehen die gegebenen Functionenelemente in die sämmtlichen verschiedenen Zweige der Functionen $u, v, w, \ldots$ über. Von diesen setzen wir voraus, dass sie sich alle in der Form

$$
c_{1} y_{1}+c_{2} y_{2}+\cdots+c_{\lambda} y_{\lambda}
$$

darstellen lassen, wo $c_{1}, c_{2}, \ldots c_{\lambda}$ Constanten sind und $y_{1}, y_{2}, \ldots y_{\lambda}$ von einander unabhängige $Z$ weige der Functionen $u, v, w, \ldots$ bezeichnen, oder, um diese im Folgenden oft wiederkehrende Bedingung kürzer auszudrricken, wir nehmen an, dass unter den verschiedenen Zweigen der Functionen $u, v, w, \ldots$ nur $\lambda$ von einander unabhängige enthalten sind. 
Setzt man nun

$$
\Sigma \pm y y_{i}^{(1)} \ldots y_{\lambda}^{(\lambda)}=D y^{(\lambda)}+D_{1} y^{(\lambda-1)}+\cdots+D_{i} y
$$

so ist $D$ als Determinante von $\lambda$ unter einander unabhãngigen Functionen von Null verschieden, und die lineare Differentialgleichung

$$
P=y^{(\lambda)}+\frac{D_{1}}{D} y^{(\lambda-1)}+\cdots+\frac{D_{\lambda}}{D} y=0
$$

hat die Functionen $y_{1}, y_{2}, \ldots y_{\lambda}$ und daher auch ihre linearen Verbindungen $u, v, w, \ldots$ zu particulären Integralen. Durchläuft $x$ irgend einen geschlossenen Weg, so verwandelt sich $y_{*}$ der Annahme nach in einen Ausdruck von der Form

$$
c_{x 1} y_{1}+c_{x 2} y_{2}+\cdots+c_{x \lambda} y_{\lambda},
$$

und daher gehen $D, D_{1}, \ldots D_{\lambda}$ nach dem Multiplicationstheorem der Determinanten in sich selbst über, multiplicirt mit der Determinante

$$
C=\Sigma \pm c_{11} c_{22} \ldots c_{\lambda \lambda} \text {. }
$$

Wenn die Constante $C$ für irgend einen der geschlossenen Wege, die $x$ durchlaufen kann, den Werth Null hälte, so würde $D$ auf dieser Linie in $C D=0$ übergehen und mithin ein $Z$ weig der Function $D$ identisch verschwinden. Dann müsste aber die analytische Function $D$ überhaupt gleich Null sein, was nicht angeht. Da also $C$ für jeden Weg einen bestimmten, von Null verschiedenen, endlichen Werth hat, so kann der Bruch $\frac{C D_{\varkappa}}{C D}$ durch $C$ gehoben werden, und folglich sind die Coefficienten der linearen Differentialgleichung $P=0$ eindeutige Functionen von $x$. Da sie aber ebenso wie $u, v, w, \ldots$ an jeder Stelle, $a$, mit einer endlichen Potenz von $x$-a (oder $\frac{1}{x}$ für $a=\infty$ ) multiplicirt, endlich bleiben, so müssen sie rationale Functionen von $x$ sein. Weil deren Nenner alle zusammen nicht unendlich oft verschwinden können, so besitzt die Differentialgleichung $P=0$ nur eine endliche Anzahl singulärer Punkte *) und hat mithin in Folge der Beschaffenheit

*) Die singulären Punkte einer linearen Differentialgleichung sind nicht identisch mit den singulären Punkten der sie befriedigenden Functionen. Die letzteren sind diejenigen Stellen, an denen die Entwickelbarkeit der Integrale, die ersteren diejenigen, an denen, falls der Coefficient der höchsten Ableitung gleich Eins ist, die Entwickelbarkeit der Coefficienten nach ganzen positiven Potenzen der Aenderung des Arguments aufhört. Zu den singulären Punkten der Differentialgleichung gehören ausser den singulären Punkten der Integrale roch die ausserwesentlich singulären Stellen. (Vergl. die Abh. des Herrn Fuchs, dieses Journal, Bd. 68, S. 378.) 
ihrer Integrale die Form

$$
y^{(\lambda)}+\frac{p_{1}}{p} y^{(\lambda-1)}+\frac{p_{2}}{p^{3}} y^{(\lambda-2)}+\cdots+\frac{p_{\lambda}}{p^{\lambda}} y=0,
$$

wo $p, p_{1}, \ldots p_{\lambda}$ ganze Functionen sind, $p$ keinen quadratischen Divisor hat, und $p_{x}$, wenn $p$ vom $\mu^{\text {ten }}$ Grade ist, den $x(\mu-1)^{\text {ten }}$ Grad nicht überschreitet. Damit ist der Satz bewiesen:

Wenn mehrere über die ganze Ebene fortsetzbare analytische Functionen nur eine endliche Anzahl singulärer Stellen haben und an jeder derselben, mit einer endlichen Potenz der Aenderung des Arguments multiplicirt, endlich bleiben, und wenn unter ihren verschiedenen Zweigen nur $\lambda$ von einander unabhängige enthalten sind, so genügen sie einer linearen Differentialgleichung $\lambda$ ter Ordnung.

Functionen der angegebenen Art sind z. B. die Wurzeln einer algebraischen Gleichung, deren Coefficienten rationale Functionen einer Variablen sind. Von diesen gilt also der Satz:

Wenn sich die Wurzeln einer algebraischen Gleichung durch $\lambda$ unter ihnen und nicht durch weniger als $\lambda$ linear mit constanten Coefficienten ausdrücken lassen, so genügen sie einer linearen Differentialgleichung $\lambda$ ter Ordnung.

Man kann umgekehrt die Frage aufwerfen, unter welchen Bedingungen eine lineare Differentialgleichung algebraische Integrale hat. Wenn eine Differentialgleichung durch eine Wurzel $y$ einer irreductibeln algebraischen Gleichung zwischen $x$ und $y$ befriedigt wird, so müssen ihr auch die sämmtlichen verschiedenen $Z$ weige der Function $y$, d. h. alle Wurzeln jener irreductibeln Gleichung genügen. Wenn diese sich durch $\mu$ von ihnen linear mit constanten Coefficienten ausdrücken lassen, so genügt $y$ einer linearen Differentialgleichung $\mu^{\text {ter }}$ Ordnung, die nur algebraische Integrale hat, während die gegebene Differentialgleichung ausserdem noch andere haben kann. Die Frage nach der Integrabilităt einer linearen Differentialgleichung durch algebraische Functionen zerlegt sich somit in die zwei Fragen, ob eine lineare Differentialgleichung mit einer andern niedrigerer Ordnung Integrale gemeinsam hat, und ob sie nur algebraische Functionen zu Integralen hat *). Und das erstere Problem ist es, auf das ich in diesem Aufsatze die Aufmerksamkeit zu lenken wünsche.

*) Vergl. Abel, oeuvres complètes, Tom. II., pag. 189. 
\$. 3 .

Wir gehen nach diesen vorbereitenden Sälzen an die Exposition des in der Einleitung definirten Begriffs einer irreductibeln linearen Differentialgleichung.

Unter den sämmtlichen Zweigen einer Function, die einer linearen Differentialgleichung $\lambda^{\text {ter }}$ Ordnung mit eindeutigen Coefficienten genügt, können nicht mehr als $\lambda$ von einander unabhängige enthalten sein. Befinden sich unter ihnen nur $\mu$ solche, wo $\mu<\lambda$ ist, so genügt die Function nach dem im $\$$. 2 entwickelten Satze einer linearen Differentialgleichung $\mu^{\text {ter }}$ Ordnung, und mithin ist die gegebene Differentialgleichung $\lambda$ ter Ordnung reductibel. Daraus folgt umgekehrt:

I. Die Anzahl der unter einander unabhängigen Zweige jedes particulären Integrals einer irreductibeln linearen Differentialgleichung ist genau gleich der Ordnung der Differentialgleichung.

Wenn aber die Differentialgleichung $\lambda^{\text {ter }}$ Ordnung reductibel ist und mit einer $\mu^{\text {ter }}$ Ordnung $(\mu<\lambda)$ ein Integral gemeinsam hat, so befinden sich unter den verschiedenen $Z$ weigen dieser Function, da sie einer linearen Differentialgleichung $\mu^{\text {ter }}$ Ordnung genügt, nicht mehr als $\mu$, jedenfalls also weniger als $\lambda$ von einander unabhängige. Daraus schliesst man:

II. Unter den Integralen einer reductibeln linearen Differentialgleichung befinden sich stets einige, unter deren verschiedenen Zweigen weniger von einander unabhängige enthalten sind, als die Ordnung der Differentialgleichung angiebt.

Wenn eine Function $y$ eine lineare Differentialgleichung befriedigt, so genügen derselben auch die sämmtlichen verschiedenen $Z$ weige dieser Function. Angenommen, $y$ ist ein Integral einer irreductibeln Differentialgleichung $\mu$ ter Ordnung, so befinden sich unter ihren verschiedenen $Z$ weigen genau $\mu$ von einander unabhängige $y_{1}, y_{2}, \ldots y_{\mu}$, durch die sich alle andern linear ausdräcken lassen. Wenn nun $y$ noch einer andern Differentialgleichung, $\lambda$ ter Ordnung, genügt, so müssen auch $y_{1}, y_{2}, \ldots y_{\mu}$ dieselbe befriedigen und folglich auch der Ausdruck $c_{1} y_{1}+c_{2} y_{2}+\cdots+c_{\mu} y_{\mu}$, in dem die Constanten $c_{1}, c_{2}, \ldots c_{\mu}$ ganz willkürlich sind, d. h., das allgemeine Integral der irreductibeln Differentialgleichung. Somit gilt der Satz:

III. Wenn eine lineare Differentialgleichung mit einer irreductibeln ein Integral gemeinsam hat, so hat sie auch alle Integrale mit ihr gemeinsam.

Wenn eine Differentialgleichung $\lambda$ ter Ordnung reductibel ist, so hat sie mit einer $\mu^{\text {ter }}$ Ordnung $(\mu<\lambda)$ ein Integral $y$ gemeinsam. Die verschiedenen 
Zweige dieser Function lassen sich durch einige von ihnen, $y_{1}, y_{2}, \ldots y_{v}$, die unter einander unabhängig sind, linear ausdrücken. Ihre Anzahl $\nu$ kann höchstens gleich $\mu$ sein. Dann genügt $y$ nach dem Satze des $\$$. 2 einer Differentialgleichung $\nu^{\text {ter }}$ Ordnung, deren allgemeines Integral $c_{1} y_{1}+c_{2} y_{2}+\cdots+c_{\nu} y_{v}$ ist. Jeder Differentialgleichung, der $y$ genügt, müssen aber auch $y_{1}, y_{2}, \ldots y_{\nu}$ genügen. Daher befriedigt $c_{1} y_{1}+c_{2} y_{2}+\cdots+c_{\nu} y_{\nu}$ die reductible Differentialgleichung $\lambda^{\text {ter }}$ Ordnung. Daraus fliesst der Satz:

IV. Wenn eine lineare Differentialgleichung reductibel ist, so giebt es eine lineare Differentialgleichung niedrigerer Ordnung, mit der sie alle Integrale gemeinsam hat.

Die letztere kann wieder reductibel sein. Dann giebt es wieder eine lineare Differentialgleichung niedrigerer Ordnung, mit der sie alle Integrale gemeinsam hat, und wenn diese noch nicht irreductibel ist, auch für sie eine. Da aber die Ordnungen dieser verschiedenen Differentialgleichungen eine abnehmende Reihe bilden, die nicht mehr als $\lambda$ Glieder enthalten kann, so schliesst man:

V. Wenn eine lineare Differentialgleichung reductibel ist, so giebt es eine oder mehrere irreductible Differentialgleichungen, mit denen sie alle Integrale gemeinsam hat.

Ich mache aber gleich darauf aufmerksam, dass es hier nicht, wie bei den reductibeln algebraischen Gleichungen stets mehrere irreductible Differentialgleichungen geben muss, mit denen eine reductible alle Integrale gemeinsam hat, und dass, wenn es mehrere giebt, die Summe ihrer Ordnungen kleiner sein kann, als die Ordnung der reductibeln Differentialgleichung.

S. 4.

Wenn die lineare Differentialgleichung $P=0$ mit der linearen Differentialgleichung niedrigerer Ordnung $Q=0$ alle Integrale gemeinsam hat, so kanń sie, da sie ausserdem Integrale hat, welche $Q$ nicht annulliren, auch singuläre Stellen haben, an denen die Nenner der Coefficienten von $Q$ nicht verschwinden. Umgekehrt kann aber auch die Differentialgleichung $Q=0$, trotzdem alle ihre Integrale die Gleichung $\boldsymbol{P}=\mathbf{0}$ befriedigen, singuläre Stellen haben, an denen die Coefficienten von $P$ nicht unendlich werden. Ist $a$ eine solche, so kann hier freilich kein Integral der Differentialgleichung $Q=0$ unstetig werden oder sich verzweigen, da sonst auch ein Integral von $P=0$ 
und folglich auch diese Differentialgleichung selbst an $a$ einen singulären Punkt hätte. Daher muss $a$ ein ausserwesentlich singulärer Punkt der Differentialgleichung,$Q=0$ sein.

Der Unterschied zwischen einem nicht singulären und einem ausserwesentlich -singulären Punkle einer Differentialgleichung $\mu^{\text {ter }}$ Ordnung $Q=0$ besteht bekanntlich darin, dass an einer nicht singulären Stelle $a$ sich $\mu$ Integrale finden lassen, deren Entwickelungen nach ganzen positiven Potenzen von $x-a$ der Reihe nach mit

$$
(x-a)^{\prime \prime}, \quad(x-a)^{1}, \cdots(x-a)^{\mu-i}
$$

anfangen, an einer ausserwesentlich singulären Stelle $a$ aber $\mu$ Integrale, deren Entwickelungen nach ganzen positiven Potenzen von $x-a$ mit

$$
(x-a)^{\rho_{1}}, \quad(x-a)^{\rho_{2}}, \quad \ldots \quad(x-a)^{\rho_{\mu}}
$$

beginnen; wo die unter einander verschiedenen positiven ganzen Zahlen $\varrho_{1}$, $\varrho_{2}, \ldots \varrho_{\mu}$ nicht mit den Zahlen $0,1, \ldots \mu-1$ übereinstimmen, also nicht sämmtlich kleiner als $\mu$ sind. Ist

$$
Q=y^{(\mu)}+q_{1} y^{(\mu-1)}+\cdots+q_{\mu} y
$$

und

$f(x, \rho)=\rho(\varrho-1) \ldots(\rho-\mu+1)+(x-a) q_{1} \varrho(\varrho-1) \ldots(\rho-\mu+2)+\cdots+(x-a)^{\mu} q_{\mu}$, so sind $\varrho_{1}, \varrho_{2}, \ldots \varrho_{\mu}$ die Wurzeln der Gleichung $\mu^{\text {ten }}$ Grades

$$
f(a, \varphi)=0 \text {, }
$$

welche Herr Fuchs die zum singulären Punkte a gehörige determinirende Fundamentalgleichung nennt. Wenn eine ihrer Wurzeln grösser als $\lambda-1$ ist, so ist $a$ auch für die Differentialgleichung $\lambda^{\text {ter }}$ Ordnung $P=0$ ein ausserwesentlich singulärer Punkt. Daraus ergiebt sich der Satz:

I. Wenn eine lineare Differentialgleichung mit einer andern niedrigerer Ordnung alle Integrale gemeinsam hat, so sind alle singulären Punkte der letzteren, welche es nicht zugleich für die erstere sind, ausserwesentlich singuläre Punkte, für welche die Wurzeln der zugehörigen determinirenden Fundamentalgleichung kleiner als die Ordnung der ersten Differentialgleichung sind.

Wenn eine Differentialgleichung reductibel ist, so giebt es eine Differentialgleichung niedrigerer Ordnung, mit welcher sie alle Integrale gemeinsam hat. Nun hat die letztere in der Umgebung einer singulären Stelle a stets solche Integrale, die bei einem Umlauf der Variablen um den Punkt $a$ in sich selbst, mit einer Constanten multiplicirt, übergehen. Daher muss eins 
derjenigen Integrale der reductibeln Differentialgleichung, welchen dieselbe Eigenschaft zukommt, einer Differentialgleichung niedrigerer Ordnung genügen. Die Anzahl dieser Integrale ist im Allgemeinen eine endliche, wenn man zwei -Integrale, deren Quotient constant ist, nicht als verschieden betrachtet. Wenn die reductible Differentialgleichung nicht gerade von dieser Regel, eine Ausnahme macht, so kann man eine bestimmte Anzahl von ihren Integralen angeben, unter welchen sich die befinden müssen, die eine Differentialgleichung niedrigerer Ordnung befriedigen. Da dieser allgemeine Fall somit eine besondere Beachtung verdient, so stellen wir uns die Aufgabe, die Bedingungen zu ermitteln, unter denen er keine Ausnahme erleidet.

Wenn ein Integral einer linearen Differentialgleichung $\lambda$ ter Ordnung bei einem Umlauf von $x$ um $a$ in sich selbst, mit $r$ multiplicirt, übergeht, so ist $r$ eine Wurzel einer bestimmten Gleichung $\lambda$ ten Grades, welche Herr Fuchs die zum singulären Punkte $a$ gehörige Fundamentalgleichung nennt. Ist $r$ eine einfache Wurzel derselben, so giebt es nur ein einziges Integral, welches bei einem Umlauf der Variablen um $a$ mit $r$ multiplicirt wird (vergl. d. Abh. d. Herrn Fuchs, dieses Journal, Bd. 66, S. 132, insbesondere das Gleichungssystem (5.)). Ist aber $r$ eine $(\varkappa+1)$ fache Wurzel, so entsprechen ihr genau $x+1$ unter einander unabhängige Integrale. Diese haben in der Umgebung von $a$ die Gestalt

$$
y=\varphi_{0}+\varphi_{1} \log (x-a)+\cdots+\varphi_{x}(\log (x-a))^{x},
$$

wo $\varphi_{0}, \varphi_{1}, \ldots \varphi_{*}$ Functionen sind, die bei einem Umlauf von $x$ um $a$ in sich selbst mit $r$ multiplicirt übergehen, und die auch zum Theil identisch verschwinden können. Der Exponent der höchsten in diesen Integralen wirklich vorkommenden Potenz von $\log (x-a)$ kann nicht grösser als $*$ sein. Wenn er gleich $\varkappa$ ist, so entspricht der Wurzel $r$ nur ein einziges Integral, in dem keine Logarithmen vorkommen.

Dem Beweise dieser Behauptung schicken wir eine allgemeine Bemerkung voraus. Ist $y_{\mu}$ ein Integral von der Form

$$
y_{\mu}=\varphi_{\mu, 0}+\varphi_{\mu, 1} \log (x-a)+\cdots+\varphi_{\mu, \mu}(\log (x-a))^{\mu},
$$

wo $\varphi_{\mu, \mu}$ von Null verschieden ist, so geht $y_{\mu}$ bei einem Umlauf von $x$ um $a$ in $y_{\mu}+2 \pi i \mu y_{\mu-1}$ über, wo

$$
y_{\mu-1}=\varphi_{\mu-1,0}+\dot{\varphi}_{\mu-1,1} \log (x-a)+\cdots+\varphi_{\mu-1, \mu-1}(\log (x-a))^{\mu-1}
$$

ist, und $\varphi_{\mu-1, \mu-1}=\varphi_{\mu, \mu}$, also yon Null verschieden ist. Da $y_{\mu-1}$ ebenfalls ein Integral der Differentialgleichung sein muss, so schliessen wir daraus: Wenn 
der Wurzel $r$ ein Integral entspricht, in welchem die höchste vorkommende Potenz von $\log (x-a)$ die $\mu^{\text {te }}$ ist, so entspricht ihr auch ein Integral, in welchem diese Potenz die $\nu^{\text {te }}$ ist, wo $\nu$ irgend eine Zahl von 0 bis $\mu-1$ sein kann.

Wenn daher der Wurzel $r$ ein Integral entspricht, in welchem die höchste auftretende Potenz von $\log (x-a)$ die $\varkappa^{\text {te }}$ ist, so entsprechen ihr $x+1$ Integrale, $y_{0}, y_{1}, \ldots y_{x}$, in denen diese Potenz der Reihe nach die $0^{\text {te }}, 1^{\text {te }}, \ldots x^{\text {te }}$ ist, und die desshalb unter einander unabhängig sind. Da einer $(x+1)$ fachen Wurzel $r$ nicht mehr als $x+1$ von einander unabhängige Integrale entsprechen, so muss sich jedes Integral, $y_{\circlearrowleft}^{\prime}$, das bei einem Umlauf von $x$ um $a$ in sich selbst mit $r$ multiplicirt, übergeht, in der Form

$$
y_{10}^{\prime} \doteq c_{0} y_{0}+c_{1} y_{1}+\cdots+c_{*} y_{*}
$$

darstellen lassen. Vergleicht man auf beiden Seiten dieser Gleichung die Coefficienten von $(\log (x-a))^{x}$, so erkennt man, dass $c_{n}=0$ ist; vergleicht man dann die von $(\log (x-a))^{x-1}$, so findet man, dass auch $c_{x-1}=0^{\circ}$ ist, u. s. w. Daher ist $y_{0}^{\prime}=c_{0} y_{0}$, also von $y_{0}$ nicht verschieden.

Damit umgekehrt die Differentialgleichung nur ein einziges Integral besitzt, welches bei einem Umlauf von $x$ um $a$ in sich selbst übergeht, multiplicirt mit der $(\varkappa+1)$ fachen Wurzel $r$ der zu a gehörigen Fundamentalgleichung, darf der Exponent der höchsten Potenz von $\log (x-a)$, welche in den der Wurzel $r$ entsprechenden Integralen vorkommt, nicht kleiner als $*$ sein. Sei, um dies zu beweisen, $y_{0}$ das Integral, das bei einem Umlaufe von $x$ um $a$ mit $r$ multiplicirt wird. Ist $x>0$, so muss die Differentialgleichung noch andere zur Wurzel $r$ gehörige Integrale haben, und da $y_{1}$ das einzige Integral seiner Art sein soll, so müssen dieselben Logarithmen enthalten. Nach der oben gemachten Bemerkung muss aber, wenn der Wurzel $r$ überhaupt Integrale mit Logarithmen entsprechen, sich unter ihnen auch eins von der Form

$$
y_{1}=\varphi_{1,0}+\varphi_{1,1} \log (x-a)
$$

befinden. Da nach einem Satze des Herrn Fuchs (dieses Journal Bd. 68, S. 356) der Coefficient der höchsten in einem Integrale auftretenden Potenz von $\log (x-a)$ ebenfalls die Differentialgleichung befriedigt, und da derselbe bei einem Umlaufe von $x$ um a mit $r$ multiplicirt wird, so muss, bei passender Verfügung über den in $y_{1}$ enthaltenen constanten Factor, $\varphi_{1,1}=y_{0}$ und daher

$$
y_{1}=\varphi_{1,0}+y_{0} \log (x-a)
$$

sein. Wenn noch ein zweites Integral existirt, in welchem die höchste vor- 
kommende Potenz von $\log (x-a)$ die erste ist, so hat es die Form

Daher ist

$$
y_{1}^{\prime}=\varphi_{1,0}^{\prime}+y_{0} \log (x-a) \text {. }
$$

$$
y_{1}^{\prime}-y_{1}=\varphi_{1,0}^{\prime}-\varphi_{1,0}=c_{0} y_{0},
$$

weil diese Differenz zweier Integrale die Differentialgleichung befriedigt und bei einem Umlauf von $x$ um $a$ mit $r$ multiplicirt wird. Jedes der Wurzel $r$ entsprechende Integral, welches $\log (x-a)$ nur in der ersten Potenz enthält, lässt sich also durch $y_{0}$ und $y_{1}$ linear ausdrücken. Wenn aber $\varkappa>1$ ist, so entsprechen der Wurzel $\boldsymbol{r}$ mehr als zwei unter einander unabhängige Integrale. In diesen mũssen also höhere Potenzen von $\log (x-a)$ auftreten, und folglich giebt es unter ihnen auch eins von der Form

Ist

$$
y_{2}=\varphi_{2,0}+\varphi_{2,1} \log (x-a)+y_{0}(\log (x-a))^{2} \text {. }
$$

$$
y_{2}^{\prime}=\varphi_{2,0}^{\prime}+\varphi_{2,1}^{\prime} \log (x-a)+y_{0}(\log (x-a))^{2}
$$

ein zweites von derselben Form, so ist

$$
y_{2}^{\prime}-y_{2}=\left(\varphi_{2,0}^{\prime}-\varphi_{2,0}\right)+\left(\varphi_{2,1}^{\prime}-\varphi_{2,1}\right) \log (x-a)=c_{0} y_{0}+c_{1} y_{1}
$$

Daher lassen sich alle Integrale, welche $\log (x-a)$ höchstens in der zweiten Potenz enthalten, durch $y_{0}, y_{1}, y_{2}$ linear ausdrücken. Indem man so weiter schliesst, erkennt man, dass wenn der Wurzel $r$ nur ein einziges Integral ohne Logarithmen entsprechen soll, die höchste Potenz von $\log (x-a)$, welche in den der Wurzel $r$ entsprechenden Integralen vorkommt, die $\varkappa^{\text {te }}$ sein muss. Wir können also den Satz aussprechen:

II. Damit eine lineare Differentialgleichung nur eine endliche Anzahl von Integralen besitzt, welche bei einem Umlauf der Variablen $x$ um eine singuläre Stelle $a$ in sich selbst, mit einer Constanten multiplicirt, übergehen, ist nothwendig und hinreichend, dass die Wurzeln der zum Punkte a gehörigen Fundamentalgleichung entweder alle unter einander verschieden sind, oder dass die höchste Potenz von $\log (x-a)$, welche in den einer $(x+1)$ fachen Wurzel entsprechenden Integralen wirklich vorkommt, die $\varkappa^{\text {te }}$ ist.

Erinnert man sich nun des Ausgangspunktes dieser ganzen Entwickelung, so gelangt man zu dem Satze:

III. Wenn die Wurzeln der Fundamentalgleichung, welche zu einer singulären Stelle a einer reductibeln linearen Differentialgleichung gehört, alle unter einander verschieden sind, oder wenn die höchste Potenz von $\log (x-a)$, welche in den einer $(x+1)$ fachen Wurzel entsprechenden Integralen vorkommt, 
die $\varkappa^{t e}$ ist, so muss eins der Integrale, welche in der Umgebung von a keine Logarithmen enthalten, und deren Anzahl höchstens gleich der Ordnung der Differentialgleichung ist, einer Differentialgleichung niedrigerer Ordnung genügen.

Wenn man nun eine Differentialgleichung $\lambda$ ter Ordnung, die einen singulären Punkt von der angegebenen Beschaffenheit besitzt, integriren kann, d. h. wenn man den Verlauf ihrer Integrale durch die ganze Ebene zu verfolgen vermag, so wird man durch diesen Satz in den Stand gesetzt, die Frage, ob sie reductibel oder irreductibel ist, zu entscheiden. Man hat nämlich nur zu ermitteln, ob sich unter den Integralen, die bei einem Umlauf von $x$ um $a$ in sich selbst, mit einer Constanten multiplicirt, übergehen, eins befindet, unter dessen verschiedenen $Z$ weigen weniger als $\lambda$ von einander unabhängige enthalten sind. Zur.Ausführung dieser Untersuchung ist nur die Kenntniss der linearen Relationen erforderlich, welche zwischen den Integralen der verschiedenen, den einzelnen singulären Punkten entsprechenden Fundamentalsysteme bestehen *).

In besonders einfacher Weise lässt sich die Bedingung dafür, dass eine gegebene Differentialgleichung $\lambda^{\text {ter }}$ Ordnung mit einer $\mu^{\text {ter }}$ Ordnung alle Integrale gemeinsam hat, angeben, wenn keine der zu den singulären Punkten gehörigen Fundamentalgleichungen mehrfache Wurzeln hat. Man verbinde eine Verzweigungsstelle $a$ mit allen andern durch Linien $L$, die durch keinen Verzweigungspunkt hindurchführen. Das bei der gemachten Annahme vollständig bestimmte System der Integrale, welche bei einem Umlauf um eine Verzweigungsstelle in sich selbst übergehen, multiplicirt mit den verschiedenen Wurzeln der zu ihr gehörigen Fundamentalgleichung, soll das diesem singulären Punkte entsprechende Fundamentalsystem genannt werden. Es seien $y_{1}, y_{2}, \ldots y_{\lambda}$ die Integrale des zum Verzweigungspunkte $a, z_{1}, z_{2}, \ldots z_{\lambda}$ die des zu einem andern $b$ gehörigen Fundamentalsystems, und es verwandle sich $y_{x}$, wenn es von $a$ längs der nach $b$ führenden Linie $L$ fortgesetzt wird, in $c_{x, 1} z_{1}+c_{x, 2} z_{2}+\cdots+c_{x, \lambda} z_{\lambda}$. Alsdann müssen unter diesen $\lambda$ linearen Ausdrücken $\mu$ sein, welche von den $\lambda$ Functionen $z_{1}, z_{2}, \ldots z_{\lambda}$ nur $\mu$ enthalten, oder es muss, bei passend gewählter Bézeichnung der Integrale, auf der Linie $L$

$$
\begin{array}{ccc}
y_{1} \text { in } c_{1,1} z_{1}+c_{1,2} z_{2}+\cdots+c_{1, \mu} z_{\mu}, \\
y_{2} \text { in } c_{2,1} z_{1}+c_{2,2} z_{2}+\cdots+c_{2, \mu} z_{\mu}, \\
\dot{y_{\mu}} \text { in } c_{\mu, 1} z_{1}+c_{\mu, 2} z_{2}+\cdots+c_{\mu, \mu} z_{\mu}
\end{array}
$$

*) Vergl. uber diese Relationen d. Abh. d. Herrn Fuchs, dieses Journal, Bd. 75 S. 212. Journal für Mathematik Bd. LXXVI. Heft 3.

32 
übergehen, während die Coefficienten $c_{1, \mu+1}, c_{1, \mu+2}, \ldots c_{1, \lambda}, c_{2, \mu+1}, \ldots c_{\mu, \lambda}$ sämmtlich verschwinden. Dass diese Bedingung für den auf den Linien $L$ auszuführenden Uebergang von $a$ zu jedem andern Verzweigungspunkte erfült wird, ist nothwendig und hinreichend, damit die gegebene Differentialgleichung $\lambda$ ter Ordnung mit einer $\mu^{\text {ter }}$ Ordnung alle Integrale gemeinsam hat.

Die Nothwendigkeit dieser Bedingung folgt aus dem oben entwickelten Satze, nach welchem, da keine der zu den Verzweigungspunkten gehörigen Fundamentalgleichungen mehrfache Wurzeln. besitzt, $\mu$ von den Integralen des jedem singulären Punkte entsprechenden Fundamentalsystems der Differentialgleichung $\mu^{\text {ter }}$ Ordnung genügen müssen. Dass, sie aber auch hinreichend ist, erkennt man, indem man jeden von einem Punkte $a^{\prime}$ in der Umgebung von $a$ ausgehenden geschlossenen Weg nach.der Puiseuxschen Methode durch eine Reihe von Elementarcontouren ersetzt, d. h. von geschlossenen Strecken, die von $a^{\prime}$ längs $\operatorname{der} a$ mit einem andern Verzweigungspunkt $b$ verbindenden Linie $L$ nach einem nahe vor $b$ liegenden Punkte $b^{\prime}$ dieses Weges, dann von $b^{\prime}$ in einem sehr kleinen Kreise um $b$ herum und zuletzt von $b^{\prime}$ längs $L$ nach $a^{\prime}$ zurückführen.

Wenn demnach keine der zwei singulären Punkten einer linearen Differentialgleichung $\lambda_{\text {ter }}$ Ordnung zugehörigen Fundamentalgleichungen mehrfache Wurzeln hat, und wenn in den linearen Relationen, welche bei einer bestimmten Verbindung dieser beiden Stellen die Integrale des der einen entsprechenden Fundamentalsystems durch die Integrale des der andern entsprechenden ausdrücken, keiner der $\lambda^{2}$ Coefficienten verschwindet, so ist die Differentialgleichung irreductibel. Dies ist auch schon der Fall, wenn sich unter den linearen Ausdrücken für die $\lambda$. Integrale des dem ersten Punkte entsprechenden Fundamentalsystems nicht $\mu$ finden lässen $(\mu=1,2, \ldots \lambda-1)$, welche nur $\mu$ von den Integralen des dem zweiten Punkte entsprechenden enthalten.

\section{\$. 5.}

Als Beispiel für die eben entwickelte Lösung des aufgestellten Problems will ich die Differentialgleichung

$$
x(1-x) y^{\prime \prime}+(\gamma-(\alpha+\beta+1) x) y-\alpha \beta y=0
$$

behandeln, der die hypergeometrische Reihe

$$
F(\alpha, \beta, \gamma, x)=1+\frac{\alpha \beta}{\gamma} x+\frac{\alpha(\alpha+1) \beta(\beta+1)}{\gamma(\gamma+1) 1.2} x^{2}+\cdots
$$

genügt. Ihre singulären Stellen sind die Punkte $0, \infty$ und 1. Damit an 
keiner derselben in den Entwickelungen der Integrale Logarithmen auftreten, nehmen wir an, dass $\gamma, \alpha+\beta-\gamma$ und $\alpha-\beta$ keine ganzen Zahlen sind. Alsdann hat keine der zu den Verzweigungspunkten gehörigen Fundamentalgleichungen mehrfache Wurzeln. Die den singulären Stellen entsprechenden Fundamentalsysteme werden gebildet ${ }^{*}$ )

für den Werth $\infty$ von den Integralen

$$
\begin{array}{lll}
v=\left(\frac{1}{x}\right)^{\alpha} F\left(\alpha, \alpha-\gamma+1, \alpha-\beta+1, \frac{1}{x}\right), & v^{\prime}=\left(\frac{1}{x}\right)^{\beta} F\left(\beta, \beta-\gamma+1, \beta-\alpha+1, \frac{1}{x}\right), \\
\quad \text { für den Werth } 0 \text { von } & u^{\prime}=x^{1-\gamma} F(\alpha-\gamma+1, \beta-\gamma+1,2-\gamma, x),
\end{array}
$$

für den Werth 1 von

$$
w=F(\alpha, \beta, \alpha+\beta-\gamma+1,1-x), \quad w^{\prime}=(1-x)^{\gamma-\alpha-\beta} F(\gamma-\alpha, \gamma-\beta, \gamma-\alpha-\beta+1,1-x) \text {. }
$$

Um diese Funclionen eifldeutig definiren zu können, schliessen wir die negative Abscissenaxe vom Gebiete der Variabeln $x$ (oder $1-x$ bei $w$ und $w^{\prime}$ ) aus, indem wir längs derselben durch die Constructionsebene einen Schnitt führen. In der so entslandenen Fläche ist die Function $\log (x)$ eindeutig definirt, wenn ihr im Punkte 1 der Werth 0 ertheilt wird, ebenso die Function $x^{n}$ auch für gebrochene und irrationale Werthe des Exponenten, wenn dafür der durch die Gleichung

$$
x^{n}=e^{n \log x}
$$

bestimmte Werth genommen wird. Für die Stelle $a$ wählen wir den Punkt 1, weil der Convergenzbereich der Reihen $w$ und $w^{\prime}$ sowohl mit dem der Reihen $u$ und $u^{\prime}$, als auch mit dem der Reihen $v$ und $v^{\prime}$ ein Stück gemeinsam hat. Aus diesem Grunde ist es hier auch unnöthig, die Stelle 1 mit den Punkten 0 und $\infty$ durch Linien zu verbinden. Zwischen den Integralen der den verschiedenen singulären Punkten entsprechenden Fundamentalsysteme bestehen dann folgende Relationen:

$$
\begin{aligned}
& w=\frac{\Pi(\alpha+\beta-\gamma) \Pi(-\gamma)}{\Pi(\alpha-\gamma)} \quad u+\frac{\Pi(\alpha+\beta-\gamma) \Pi(\gamma-2)}{\Pi(\beta-\gamma)} \quad u^{\prime}, \\
& w^{\prime}=\frac{\Pi(\gamma-\alpha-\beta) \Pi(-\gamma)}{\Pi(-\alpha)} \quad u+\frac{\Pi(\gamma-\alpha-\beta) \Pi(\gamma-2)}{\Pi(-\beta)} u^{\prime}, \\
& w=\frac{\Pi(\alpha+\beta-\gamma) \Pi(\beta-\alpha-1)}{\Pi(\beta-\gamma) \Pi(\beta-1)} v+\frac{\Pi(\alpha+\beta-\gamma) \Pi(\alpha-\beta-1)}{\Pi(\alpha-\gamma) \Pi(\alpha-1)} v^{\prime}, \\
& w^{\prime}=\frac{\Pi(\gamma-\alpha-\beta) \Pi}{\Pi(-\alpha)} \frac{(\beta-\alpha-1)}{\Pi(\gamma-\alpha-1)} v+\frac{\Pi(\gamma-\alpha-\beta) \Pi(\alpha-\beta-1)}{\Pi(-\beta)} v^{\prime} .
\end{aligned}
$$

*) Die hier benutzten Eigenschaften der hypergeometrischen Reihe findet man in der Abhandlung des Herrn Kummer, dieses Journal, Bd. 15, S. 52-60 und in Gauss' Werken, Bd. 3, S. 207-220. 
Diese Gleichungen sind für alle Punkte der Flächenstücke gültig, welche den Convergenzbereichen der auf ihrer rechten und auf ihrer linken Seite stehenden Reihen gemeinsam sind.

Damit die gegebene Differentialgleichung zweiter Ordnung reductibel ist, also mit einer erster Ordnung ein Integral gemeinsam hat, muss entweder in jedem der beiden Ausdrücke für $w$ einer der Coefficienten verschwinden oder in jedem der beiden Ausdrücke für $w^{\prime}$. Da $\Pi(x)$ für keinen endlichen Werth von $x$ verschwindet und für alle negativen ganzen Zahlen unendlich gross wird, so zeigt der blosse Anblick der obigen Gleichungen, dass diese Bedingung erfüllt ist, wenn entweder $\alpha(\operatorname{oder} \beta)$ oder $\gamma-\alpha(\operatorname{oder} \gamma-\beta)$ eine ganze Zahl ist. Bedeutet $\nu$ eine positive ganze Zahl oder Null, so ist demnach die gegebene Differentialgleichung in folgenden acht Fällen reductibel:

1) $\alpha=\nu+1$. In Folge der bekannten Relation

$$
F(\alpha, \beta, \gamma, x)=(1-x)^{\gamma-\alpha-\beta} F(\gamma-\alpha, \gamma-\beta, \gamma, x)
$$

ist das Integral

$u^{\prime}=x^{1-\gamma} F(\alpha-\gamma+1 ; \beta-\gamma+1,2-\gamma, x)=x^{1-\gamma}(1-x)^{\gamma-\alpha-\beta} F(1-\alpha, 1-\beta, 2-\gamma, x)$.

Da aber $1-\alpha=-\nu$ ist, so bricht die Reihe $F(1-\alpha, 1-\beta, 2-\gamma, x)$ ab und stellt eine ganze Function $\nu^{\text {ten }}$ Grades dar. Die Gleichung

$$
F(1-\alpha, 1-\beta, 2-\gamma, x)=0
$$

hat, wie man aus der linearen Differentialgleichung zweiter Ordnung ersehen kann, der ihre linke Seite genügt, keine mehrfachen Wurzeln. Daher ist

$$
u^{\prime}=C x^{1-\gamma}(1-x)^{\gamma-\alpha-\beta}\left(x-a_{1}\right)\left(x-a_{2}\right) \ldots\left(x-a_{\nu}\right),
$$

wo die Grössen $0,1, a_{1}, a_{2}, \ldots a_{v}$ sämmtlich unter einander verschieden sind. Die Stellen $a_{1}, a_{2}, \ldots a_{1}$, sind die ausserwesentlich singulären Punkte der Differentialgleichung erster Ordnung, welche von den Integralen $u^{\prime}, v^{\prime}$ und $w^{\prime}$, die sich nur um constante Factoren unterscheiden, befriedigt wird. Die Exponenten, zu denen das Integral der linearen Differentialgleichung erster Ordnung in den Umgebungen dieser Punkte gehört, sind dem oben entwickelten Satze gemäss kleiner als die Ordnung der reductibeln Differentialgleichung, also sämmtlich gleich 1 .

2) $\alpha=-\nu$. In diesem Falle ist

$$
\boldsymbol{u}=\boldsymbol{F}(\alpha, \beta, \gamma, \boldsymbol{x})
$$

eine ganze Function $\nu^{\text {ten }}$ Grades, und die lineare Differentialgleichung erster Ordnung, der $u, v$ und $w$ genügen, hat nur ausserwesentlich singuläre Stellen. 
3) $\gamma-\alpha=\nu+1$. In diesem Falle ist

$$
u^{\prime}=x^{1-\gamma} F(\alpha-\gamma+1, \beta-\gamma+1,2-\gamma, x)
$$

nebst $v$ und $w$ das Integral der Differentialgleichung erster Ordnung.

4) $\gamma-\alpha=-\nu$. In diesem Falle genügt

$$
w^{\prime}=(1-x)^{\gamma-\alpha-\beta} F(\gamma-\alpha, \gamma-\beta, \gamma-\alpha-\beta+1,1-x)
$$

nebst $u$ und $v^{\prime}$ der Differentialgleichung erster Ordnung.

Durch die Vertauschung von $\alpha$ mit $\beta$, bei welcher die Differentialgleichung ungeändert bleibt, ergeben sich daraus die übrigen vier Fälle. Die ermittelten Bedingungen lassen sich folgendermassen zusammenfassen:

I. Damit die Gausssche Differentialgleichung reductibel sei, ist nothwendig und hinreichend, dass eine der hypergeometrischen Reihen mit dem vierten Elemente $x, 1-x, \frac{1}{x}, \frac{1}{1-x}, \frac{x-1}{x}, \frac{x}{x-1}$, welche, mit einer Potenz von $x$ oder 1-x multiplicirt, ïh genügen, nur aus einer endlichen Anzahl von Gliedern besteht.

Um die Bedeutung dieser Bedingung in ein helleres Licht zu setzen, wollen wir als ein zweites Beispiel die allgemeine Differentialgleichung zweiter Ordnung mit drei singulären Punkten hehandeln, dabei aber einen andern Weg einschlagen, der die Kenntniss der Relationen, die zwischen den Integralen der den verschiedenen Verzweigungspunkten entsprechenden Fundamentalsysteme bestehen, nicht voraussetzt. Diese Differentialgleichung, welche zuerst von Riemann (Beiträge zur Theorie der durch die Gausssche Reihe $F(\alpha, \beta, \gamma, x)$ darstellbaren Functionen) vollständig integrirt ist, besitzt die merkwürdige Eigenschaft, dass ihre Constanten vollständig bestimmt sind, wenn die Exponenten gegeben sind, zu welchen die Integrale der den sin- gulären Punkten entsprechenden Fundamentalsysteme gehören. (Vergl. die Abh. des Herrn Fuchs, dieses Journal, Bd. 66, S. 160.) Durch eine lineare Transformation der unabhängigen Veränderlichen kann man die drei singulären Stellen stets nach den Punkten $0, \infty$ und 1 verlegen. Sind dann $\alpha$ und $\alpha^{\prime}$ die Wurzeln der zu $0, \beta$ und $\beta^{\prime}$ die der zu $\infty, \gamma$ und $\gamma^{\prime}$ die der zu. 1 gehörigen determinirenden Fundamentalgleichung, so hat die Differentialgleichung die Gestalt

$$
\begin{aligned}
x^{2}(1-x)^{2} y^{\prime \prime}-\left(\left(\alpha+\alpha^{\prime}-1\right)+\right. & \left.\left(\beta+\beta^{\prime}+1\right) x\right) x(1-x) y^{\prime} \\
& +\left(\alpha \alpha^{\prime}+\left(\gamma \gamma^{\prime}-\alpha \alpha^{\prime}-\beta \beta^{\prime}\right) \dot{x}+\beta \beta^{\prime} x^{2}\right) y=0 .
\end{aligned}
$$

Die Summe der Wurzeln der zu den singulären Punkten gehörigen deter- 
minirenden Fundamentalgleichungen ist nach einem Satze des Herrn Fuchs (dieses Journal Bd. 66 S. 142 und 145) bei einer Differentialgleichung $\lambda^{\text {ter }}$ Ordnung mit $\mu$ endlichen singulären Punkten gleich $(\mu-1) \frac{\lambda(\lambda-1)}{2}$. Daher muss

$$
\alpha+\alpha^{\prime}+\beta+\beta^{\prime}+\gamma+\gamma^{\prime}=1
$$

sein. Ein Integral einer linearen Differentialgleichung erster Ordnung geht auf jedem geschlossenen Wege in sich selbst, mit einer Constanten multiplicirt, über. Daher kann eine lineare Verbindung der beiden Integrale des zu einer singulären Stelle gehörigen Fundamentalsystems mit zwei Coefficienten, die beide von Null verschieden sind, nicht eine Differentialgleichung erster Ordnung befriedigen, es müsste denn die Differenz der beiden Exponenten eine ganze Zahl sein und trotzdem die Entwicklungen der Integrale keine Logarithmen enthalten. Wenn also die gegebene Differentialgleichung reductibel ist, so erkennt man daraus, dass, möge jene Differenz eine ganze Zahl sein oder nicht, stets das ihrer Integrale, welches einer Differentialgleichung erster Ordnung genügt; zu einem der beiden dem singulären Punkte entsprechenden Exponenten gehören muss. Die Bezeichnung der Wurzeln der zu den singulären Punkten gehörigen determinirenden Fundamentalgleichungen sei so gewählt, dass das der Differentialgleichung erster Ordnung genügende Integral in den Umgebungen der Punkte $0, \infty, 1$ zu den Exponenten $\alpha, \beta, \gamma$ gehört.

Die allgemeine Form einer Differentialgleichung erster Ordnung ist

$$
y^{\prime}-\left(\frac{\alpha_{1}}{x-a_{1}}+\frac{\alpha_{2}}{x-a_{2}}+\cdots+\frac{\alpha_{\mu}}{x-a_{\mu}}\right) y=0
$$

die ihres Integrals also

$$
y=C\left(x-a_{1}\right)^{\alpha_{1}}\left(x-a_{2}\right)^{\alpha_{3}} \ldots\left(x-a_{\mu}\right)^{\alpha_{\mu}} .
$$

Ihre singulären Stellen sind die Punkte $a_{1}, a_{2}, \ldots a_{\mu}, \infty$. Ist $\alpha_{0}$ der Exponent, der zum Punkte $\infty$ gehört, so ist

$$
\alpha+\alpha_{1}+\cdots+\alpha_{\mu}=0 .
$$

Die Differentialgleichung erster Ordnung, mit der die gegebene ein Integral gemeinsam hat, kann ausser den Punkten $0, \infty$ und 1 nur noch ausserwesentlich singuläre Stellen haben, deren Exponenten kleiner als 2, also gleich 1 sein müssen. Daher hat ihr Integral die Gestalt

$$
y=C x^{\alpha}(1-x)^{\gamma}\left(x-a_{1}\right)\left(x-a_{2}\right) \ldots\left(x-a_{v}\right),
$$

wo die Grössen $0,1, a_{1}, \ldots a_{\nu}$ alle unter einander verschieden sind. 
Da zum Punkte $\infty$ der Exponent $\beta$ gehört, und die Summe aller Exponenten gleich Null sein muss, so ist

und daher

$$
\alpha+\beta+\gamma+\nu=0
$$

$$
\alpha+\beta+\gamma=-\nu, \quad \alpha^{\prime}+\beta^{\prime}+\gamma^{\prime}=\nu+1 .
$$

Dies ist also die nothwendige Bedingung dafür, dass die gegebene Differentialgleichung reductibel ist. Um zu zeigen, dass sie auch hinreichend ist, machen wir die Substitution

$$
y=x^{\alpha}(1-x)^{\gamma} z
$$

Dann erhält man für $z$ eine lineare Differentialgleichung zweiter Ordnung mit den drei singulären Stellen $0, \infty$ und 1. Sind $a$ und $a^{\prime}, b$ und $b^{\prime}, c$ und $c^{\prime}$ die zugehörigen Exponenten, so ist

und da ausserdem

$$
a+b+c=-\nu, \quad a^{\prime}+b^{\prime}+c^{\prime}=\nu+1,
$$

$$
a=0, \quad a^{\prime}=\alpha^{\prime}-\alpha, \quad c=0, \quad c^{\prime}=\gamma^{\prime}-\gamma,
$$

ist, so muss

$$
b=-\nu, \quad b^{\prime}=\beta^{\prime}-\beta-\nu
$$

sein. Daher lautet die Differentialgleichung, der $z$ genügt,

$$
x(1-x) z^{\prime \prime}+\left(\left(\alpha-\alpha^{\prime}+1\right)-\left(-\nu+\beta^{\prime}-\beta-\nu+1\right) x\right) z^{\prime}-(-\nu)\left(\beta^{\prime}-\beta-\nu\right) z=0 .
$$

Ihr genügt das Integral (vergl. d. Abh. von Riemann, Art. VIII. S. 21)

$$
u=F\left(-\nu, \beta^{\prime}-\beta-\nu, \alpha-\alpha^{\prime}+1, x\right),
$$

also eine ganze Function ohne quadratischen Theiler, die in den Punkten 0 und 1 nicht verschwindet, wenn nicht etwa $\alpha^{\prime}-\alpha$ eine positive ganze Zahl ist.

In diesem Falle genügt ihr die ganze Function

$$
v=x^{\nu} F\left(-\nu, \alpha^{\prime}-\alpha-\nu, \beta-\beta^{\prime}+1, \frac{1}{x}\right),
$$

wenn nicht auch $\beta^{\prime}-\beta$ eine positive ganze Zahl ist. Alsdann wird sie befriedigt von der ganzen Function

$$
w=F\left(-\nu, \beta^{\prime}-\beta-\nu, \gamma-\gamma^{\prime}+1,1-x\right),
$$

wenn nicht auch $\gamma^{\prime}-\gamma$ eine positive ganze Zahl ist. Da die Summe der drei Differenzen $\alpha^{\prime}-\alpha, \beta^{\prime}-\beta$ und $\gamma^{\prime}-\gamma$ gleich $2 \nu+1$ ist, so muss, wenn sie alle positiv und grösser als Null sind, wenigstens eine nicht grösser als $\nu$ sein.

Ist dies $\alpha^{\prime}-\alpha$, so ist

$$
u^{\prime}=x^{\alpha^{\prime}-\alpha} F\left(\alpha^{\prime}-\alpha-\nu, \gamma-\gamma^{\prime}+\nu+1, \alpha^{\prime}-\alpha+1, x\right),
$$


ist es $\beta^{\prime}-\beta$, so ist

ist es $\gamma^{\prime}-\gamma$, so ist

$$
v^{\prime}=x^{\nu+\beta^{\prime}-\beta} F\left(\beta^{\prime}-\beta-\nu, \gamma-\gamma^{\prime}+\nu+1, \beta^{\prime}-\beta+1, \frac{1}{x}\right),
$$

$$
w^{\prime}=(1-x)^{\gamma^{\prime}-\gamma} F\left(\gamma^{\prime}-\gamma-\nu, \alpha-\alpha^{\prime}+\nu+1, \gamma^{\prime}-\gamma+1,1-x\right)
$$

eine ganze Function, welche die Differentialgleichung befriedigt.

Wir gelangen also zu dem Resultate:

II. Damit die allgemeine (Riemannsche) Differentialgleichung zweiter Ordnung mit drei singulären Punkten reductibel sei, ist es nothwendig und hinreichend, dass die Summe dreier Exponenten, unter denen sich von jedem singulären Punkte einer befindet, gleich einer ganzen Zahl ist.

\section{S. 6.}

Die in $\$ .3$ entwickelten Sätze, welche die Analogie zwischen den irreductibeln algebraischen Gleichungen und den irreductibeln linearen Differentialgleichungen ins Licht setzen, sind nach einer Methode bewiesen, welche sich bei algebraischen Gleichungen nur in dem speciellen Falle anwenden lässt, wenn ihre Coeffictenten rationale Functionen einer Veränderlichen sind. Wir wollen sie jetzt auf einem anderen Wege herleiten, der mit dem bei algebraischen Gleichungen "üblichen grössere Aehnlichkeit hat und weitere Aufschlüsse über den Charakter der reductibeln Differentialgleichungen giebt (Vergl. Libri, dieses Journal, Bd. 10, S. 193, sowie die Abhandl. des Herrn Brassinne im Anhange von Sturm, Cours d'analyse (tome II., Note III.)).

Sind

und

$$
\boldsymbol{P}=y^{(\lambda)}+p_{1} y^{(\lambda-1)}+\cdots+p_{\lambda} y
$$

$$
Q=y^{(\mu)}+q_{1} y^{(\mu-1)}+\cdots+q_{\mu} y
$$

zwei Differentialausdrücke, und ist $\lambda \geqq \mu$ und $\lambda-\mu=\nu$, so kann man $P$, wie leicht zu sehen, auf die Form

$$
P=Q^{(v)}+r_{1} Q^{(\nu-1)}+\cdots+r_{\nu} Q+r_{10} R
$$

bringen, wo $Q^{(x)}$ die $\varkappa^{\text {te }}$ Ableitung von $Q$ nach $x, r_{x}$ eine rationale Function von $x$, die auch Null sein kann, und $R$ einen Differentialausdruck bedeutet, dessen Ordnung $\varrho<\mu$ ist, und in welchem der Coefficient der höchsten $\mathbf{A b}$ leitung von $y$ gleich 1 ist. Aus dieser Gleichung folgt, dass alle gemeinsamen Integrale der beiden Differentialgleichungen $P=0$ und $Q=0$ auch $R$ 
annulliren. Da wir Gleichungen von derselben Gestalt noch mehrfach benutzen werden, so schreiben wir sie in der abgekürzten Form

$$
P \equiv R(\bmod Q)
$$

In einer solchen Congruenz bedeuten $P, \dot{Q}$ und $R$ Differentialausdrücke, in denen der Coefficient der höchsten Ableitung von $y$ gleich 1 ist, und deren Ordnungen $\lambda, \mu$, $\rho$ den Ungleichheiten

$$
\lambda \geqq u>0
$$

genügen. Wenn alle Integrale der Differentialgleichung $Q=0$ auch $P$ annulliren, so folgt aus der Congruenz

$$
P \equiv R(\bmod Q)
$$

dass sie auch sämmtlich die Differentialgleichung $R=0$ befriedigen. Demnach hat die Differentialgleichung $\rho^{\text {ter }}$ Ordnung $R=0 \mu$ von einander unabhängige Integrale. Eine lineare Differentialgleichung mit eindeutigen Coefficienten kann aber nicht mehr von einander unabhängige Integrale besitzen als ihre Ordnung angiebt, ohne identisch zu verschwinden. (Vergl. \$. 1.) Daraus folgt:

I. Wenn die Differentialgleichung $P=0$ mit der Differentialgleichung $Q=0$ alle Integrale gemeinsam hat, so lässt sich $P$ in der Form

$$
P=Q^{(\nu)}+r_{1} Q^{(\nu-1)}+\cdots+r_{\nu} Q
$$

darstellen, oder es ist

$$
P \equiv \mathbf{0}(\bmod Q)
$$

Wenn die Differentialgleichung $\boldsymbol{P}=\mathbf{0}$ mit der irreductibeln Differentialgleichung $Q=0$ ein Integral $y$ gemeinsam hat, so ist die Ordnung von $Q$ nicht höher als die von $P$. Ist dann

$$
P \equiv \boldsymbol{R}(\bmod Q),
$$

so genügt $y$ auch der Differentialgleichung $R=0$. Da aber die irreductible Differentialgleichung $Q=0$ mit der Differentialgleichung niedrigerer Ordnung $\boldsymbol{R}=\mathbf{0}$ kein Integral gemeinsan haben kann, so muss $\boldsymbol{R}$ identisch verschwinden und daher

sein. Somit gilt der Satz:

$$
P \equiv \mathbf{0}(\bmod Q)
$$

II. Wenn eine lineare Differentialgleichung mit einer irreductibeln ein Integral gemeinsam hat, so hat sie auch alle Integrale mit ihr gemeinsam.

Sind $P$ und $P_{1}$ zwei Differentialausdrücke von den Ordnungen $\lambda$ und $\lambda_{1}$, so kann man die Differentialgleichung, der die gemeinsamen Integrale der 
beiden Differentialgleichungen $P=0$ und $P_{1}=0$ genügen, nach der Methode des grössten gemeinsamen Divisors bestimmen. Dazu dienen, wenn $\lambda \geqq \lambda_{1}$ ist, die Congruenzen

$$
\begin{aligned}
& P=P_{2} \quad\left(\bmod P_{1}\right), \\
& P_{1} \equiv P_{3} \quad\left(\bmod P_{2}\right), \\
& \dot{P}_{\mu-1} \equiv \dot{P}_{\mu+1}\left(\bmod P_{\mu}\right) .
\end{aligned}
$$

Ist $\lambda_{x}$ die Ordnung von $\boldsymbol{P}_{x}$, so ist $\lambda \geqq \lambda_{1}>\lambda_{2}>\cdots$, und daher muss $\lambda_{x}$ spätestens für $\varkappa=1+\lambda_{1}$ verschwinden. Ist $\lambda_{\mu+1}=0, \lambda_{\mu}$ aber von Null verschieden, so ist $\boldsymbol{P}_{\mu+1}$ entweder gleich $y$ oder gleich Null. Im ersten Falle werden die beiden Differentialgleichungen gemeinsam nur durch $y=0$ befriedigt, d. h., sie haben kein Integral mit einander gemeinsam. Im andern Falle müssen alle gemeinsamen Integrale von $P=0$ und $P_{1}=0$ auch die Differentialgleichung $\boldsymbol{P}_{\mu}=0$ befriedigen und alle Integrale der letzteren auch den beiden ersteren genügen. Daraus folgt der Satz:

III. Wenn eine lineare Differentialgleichung reductibel ist, so giebt es eine lineare Differentialgleichung niedrigerer Ordnung, mit der sie alle Integrale gemeinsam hat.

Ist nun $P=0$ eine reductible Differentialgleichung, und $Q=0$ eine Differentialgleichung niedrigerer Ordnung, mit der sie alle Integrale gemeinsam hat, so ist

$$
P \equiv 0 \quad(\bmod Q)
$$

Die linke Seite jeder reductibeln Differentialgleichung bat daher die Form

$$
P=Q^{(v)}+r_{1} Q^{(\nu-1)}+\cdots+r_{\nu} Q .
$$

Bisher haben wir mit dem Buchstaben $P$ einen Differentialausdruck bezeichnet. Jetzt soll derselbe als Operationssymbol benutzt werden. Wir schreiben nämlich

$$
y^{(\lambda)}+p_{1} y^{(\lambda-1)}+\cdots+p_{\lambda} y=P(y)
$$

so dass $P$ eine an der Function $y$ auszuführende Operation bezeichnet, nämlich

$$
\boldsymbol{P}(y)=\left(\frac{d^{\lambda}}{d x^{\lambda}}+p_{1} \frac{d^{\lambda-1}}{d x^{\lambda-1}}+\cdots+p_{\lambda}\right)(y) .
$$

Die Bedingung dafür, dass die Differentialgleichung $P(y)=0$ mit $Q(y)=0$ alle Integrale gemeinsam hat, die bisher durch die Congruenz $P \equiv \mathbf{0}(\bmod Q)$ ausgedrückt warde, kann jetzt auch in der Form

$$
P(y)=R(Q(y))
$$


geschrieben werden, wo die Operation

$$
R(y)=\left(\frac{d^{\nu}}{d x^{\nu}}+r_{1} \frac{d^{\nu-1}}{d x^{\nu-1}}+\cdots+r_{\nu}\right)(y)
$$

an dem Differentialausdruck $\mu^{\text {ter }}$ Ordnung $Q(y)$ zu vollziehen und $\lambda=\mu+\nu$ ist.

Ist $w$ das allgemeine Integral der Differentialgleichung $R(y)=0$ und $u$ ein Integral der Differentialgleichung $Q(y)=w$, so annullirt die Function $u$ auch $\boldsymbol{P}(\boldsymbol{y})$, hat also nur eine endliche Anzahl singulärer Stellen und ist an jeder derselben, $a$, mit einer endlichen Potenz von $x-a$ (oder $\frac{1}{x}$ für $a=\infty$ ) multiplicirt, endlich. Dasselbe gilt demnach auch von der Function $w=Q(u)$, und mithin hat auch $R(y)=0$ die Form, welche hier stets für die linearen Differentialg「eichungen vorausgesetzt wird.

Die Differentialgleichung $Q(y)=w$ mit den $\nu$ in $w$ enthaltenen willkürlichen Constanten ist also für die reductible Differentialgleichung als Integralgleichung zu betrachten, aus der man jene durch Differentiation und Elimination der willkürlichen Constanten herleiten kann.

Wir können demnach das Resultat aussprechen:

IV. Wenn eine lineare Differentialgleichung $\lambda^{\text {ter }}$ Ordnung mit einer $\mu^{\text {ter Ordnung }} Q(y)=0$ alle Integrale gemeinsam hat, so genügt jedes ihrer Integrale einer Differentialgleichung von der Form $Q(y)=w$, in velcher wo ein Integral einer bestimmten Differentialgleichung $(\lambda-\mu)^{\text {ter }}$ Ordnung ist.

Umgekehrt ist jede lineare Differentialgleichung reductibel, wenn sie durch eine Differentialgleichung von der Form $Q(y)=w$ integrirt wird. Diese Gestalt der Integralgleichungen ist die charakteristische Eigenschaft der reductibeln linearen Differentialgleichungen.

\section{\$. 7.}

Jeder lineare Differentialausdruck

$$
P(y)=y^{(i)}+p_{1} y^{(\lambda-1)}+\cdots+p_{i} y
$$

lässt sich auf die Form

bringen, wo

$$
P(y)=R(Q(y))
$$

$$
Q(y)=y^{(\mu)}+q_{1} y^{(u-1)}+\cdots+q_{\mu} y
$$

und

$$
\boldsymbol{R}(\boldsymbol{y})=y^{(v)}+r_{1} y^{(\nu-1)}+\cdots+r_{\nu} y
$$


zwei lineare Differentialausdrücke sind und $\lambda=\mu+\nu$ ist, vorausgesetzt, dass man die Bedingung fallen lässt, dass die Coefficienten von $Q(y)$ und $R(y)$ rationale Functionen sein sollen. Indem man die Coefficienten derselben $\mathbf{A b}-$ leitungen von $y$ auf beiden Seiten der Gleichung $P(y)=R(Q(y))$ einander gleich setzt, erhält man zur Bestimmung der $\lambda$ unbekannten Functionen $q_{1}$, $q_{2}, \ldots q_{\mu}, r_{1}, r_{2}, \ldots r_{v}$ ebenso viele Differentialgleichungen. Damit dann die Differentialgleichung $\boldsymbol{P}(y)=0$ mit einer $\mu^{\text {ter }}$ Ordnung von derselben Form alle Integrale gemeinsam hat, muss es möglich sein, diesen $\lambda$ Differentialgleichungen durch $\lambda$ rationale Functionen zu genügen.

Wir wollen auf dem angedeuteten Wege die Bedingung dafür ermitteln, dass die Differentialgleichung $\lambda^{\text {ter }}$ Ordnung $P(y)=0$ mit einer $(\lambda-1)^{\text {ter }}$ Ordnung alle Integrale gemeinsam hat. $Z u$ dem Zwecke ist es zunächst erforderlich, die $\lambda$ Functionen $q_{1}, q_{2}, \ldots q_{\lambda-1}$ und $r$ so zu bestimmen, dass die Gleichung

$$
\begin{gathered}
y^{(\lambda)}+p_{1} y^{(\lambda-1)}+\cdots+p_{\lambda} y \\
=\frac{d}{d x}\left(y^{(\lambda-1)}+q_{1} y^{(\lambda-2)}+\cdots+q_{\lambda-1} y\right)+r\left(y^{(\lambda-1)}+q_{1} y^{(\lambda-2)}+\cdots+q_{\lambda-1} y\right)
\end{gathered}
$$

zu einer identischen wird. Setzt man

$$
z=e^{\int r d x}, \quad z_{1}=q_{1} z, \ldots z_{\lambda-1}=q_{\lambda-1} z,
$$

so nimmt dieselbe die Form

$$
z\left(y^{(\lambda)}+p_{1} y^{(\lambda-1)}+\cdots+p_{\lambda} y\right)=\frac{d}{d x}\left(z y^{(\lambda-1)}+z_{1} y^{(\lambda-2)}+\cdots+z_{\lambda-1} y\right)
$$

an, aus der man erkennt, dass $z$ der Multiplicator (integrirende Factor) der Differentialgleichung $\boldsymbol{P}(\boldsymbol{y})=0$ ist. (Vergl. die Abh. des Herrn Thomé, dieses Journal, Bd. 75, S. 272.) Zur Bestimmung von $z_{x}$ erhält man die Gleichung

$$
z p_{x}=\frac{d z_{x-1}}{d x}+z
$$

welche, wenn $z_{\lambda}=0$ gesetzt wird, für $\varkappa=1,2, \ldots \lambda$ gültig ist. Daraus ergiebt sich

$$
\text { (1.) } \quad z_{x}=z p_{x}-\frac{d\left(z p_{x-1}\right)}{d x}+\frac{d^{2}\left(z p_{x-2}\right)}{d x^{2}}-\cdots+(-1)^{x} \frac{d^{x} z}{d x^{x}} .
$$

Da $z_{\lambda}=0$ ist, so genügt $z$ der linearen Differentialgleichung

$$
\text { (2.) } \quad P^{\prime}(z)=\frac{d^{\lambda} z}{d x^{\lambda}}-\frac{d^{\lambda-1}\left(z p_{1}\right)}{d x^{\lambda-1}}+\cdots+(-1) z p_{\lambda}=0 \text {. }
$$

Damit die gegebene Differentialgleichung $\lambda$ ter Ordnung $\boldsymbol{P}(\boldsymbol{y})=\mathbf{0}$ mit einer $(\lambda-1)^{\text {ter }}$ Ordnung von derselben Form alle Integrale gemeinsam hat, müssen 
$\frac{z^{\prime}}{z}, \frac{z_{1}}{z}, \ldots \frac{z_{\lambda-1}}{z}$ rationale Functionen sein. In Folge der Relation

$$
\frac{z_{x}}{z}=p_{x}-\frac{d}{d x}\left(\frac{z_{x-1}}{z}\right)-\frac{z_{x-1}}{z} \cdot \frac{z^{\prime}}{z}
$$

sind diese Bedingungen sämmtlich erfüllt, wenn $\frac{z^{\prime}}{z}$ eine rationale Function ist, oder genauer, wenn z einer linearen Differentialgleichung erster Ordnung von der hier vorausgesetzten Form genügt. Damit also eine lineare Differentialgleichung $\lambda^{\text {ter }}$ Ordnung mit einer $(\lambda-1)^{\text {ter }}$ Ordnung alle Integrale gemeinsam habe, ist nothwendig und hinreichend, dass die lineare Differentialgleichung

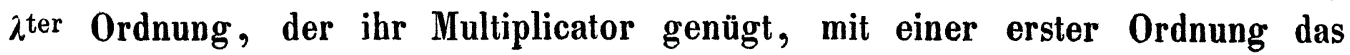
Integral gemeinsam hat. Dieses Theorem ist ein specieller Fall des allgemeineren Satzes:

I. Wenn eine lineare Differentialgleichung $\lambda$ ter Ordnung mit einer

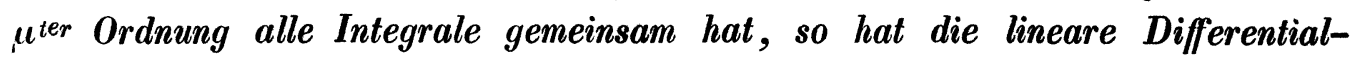
gleichung iter Ordnung, der ihr Multiplicator genügt, mit einer $(\lambda-\mu)$ ter Ordnung alle Integrale gemeinsam.

Ehe wir denselben beweisen, wollen wir kurz an die Eigenschaften der Multiplicatoren einer linearen Differentialgleichung erinnern. $\mathrm{Zu}$ dem Zwecke sehen wir vorläufig von der Differentialgleichung (2.) ab, verstehen unter $z_{i}$ eine unbestimmte Function von $x$, unter $z_{x}$ den durch die Gleichung (1.) definirten linearen Differentialausdruck $\varkappa^{\text {ter }}$ Ordnung und setzen

$$
\text { (3.) } \quad P(y, z)=z y^{(\lambda-1)}+z_{1} y^{(\lambda-2)}+\cdots+z_{\lambda} y \text {. }
$$

Dieser Differentialausdruck, welcher zwei unbestimmte Functionen, $y$ und $z$, nebst ihren Ableitungen bis zur $(\lambda-1)^{\text {ten }}$ Ordnung enthält, ist sowohl in Bezug auf $y, y^{(1)}, \ldots y^{(\lambda-1)}$, als auch in Bezug auf $z, z^{(1)}, \ldots z^{(\lambda-1)}$ eine homogene lineare Function, in welcher der Coefficient von $y^{(\lambda-1)}$ gleich $z$ und der von $z^{(\lambda-1)}$ gleich $(-1)^{\lambda-1} y$ ist. Seine charakteristische Eigenschaft besteht darin, dass, wenn $v$ irgend ein Integral der Differentialgleichung $P^{\prime}(z)=0$ bedeutet,

$$
\text { (4.) } \quad v P(y)=\frac{d}{d x} P(y, v)
$$

ist. Mithin wird die Differentialgleichung

$$
\frac{d}{d x} P(y, z)-z P(y)=0,
$$

welche in Bezug auf $z$ von der $\lambda$ ten Ordnung ist, und in welcher der Coefficient von $z^{(\lambda)}$ gleich $(-1)^{\lambda-1} y$ ist, durch alle Integrale $v$ der Differentialgleichung $P^{\prime}(z)=0$ befriedigt. 
Zwei lineare Differentialgleichungen $\lambda$ ter Ordnung, welche dieselben Integrale haben, und in denen die Coefficienten der $\lambda^{\text {ten }}$ Ableitungen der unbekannten Functionen übereinstimmen, müssen aber identisch sein, da ihre Differenz eine lineare Differentialgleichung $(\lambda-1)^{\text {ter }}$ Ordnung ist, die $\lambda$ von einander unabhängige Integrale besitzt.

Daher ist die lìnke Seite der eben aufgestellten Differentialgleichung mit $(-1)^{\lambda-1} y P^{\prime}(z)$ identisch, oder es ist

(5.) $\quad \frac{d}{d x} \dot{P}(y, z)=z P(y)-(-1)^{\lambda} y P^{\prime}(z)$.

Setzt man in dieser Identität für $y$ irgend ein Integral $u$ der Differentialgleichung $P(y)=0$, so erhält man

$$
(-1)^{\lambda-1} u P^{\prime}(z)=\frac{d}{d x} P(u, z),
$$

entsprechend dem bekannten Satze, dass die Multiplicatoren der Differentialgleichung $P^{\prime}(z)=0$ die Integrale der ursprünglichen Differentialgleichung $P(y)=0$ sind.

Nach diesen Vorbereitungen kehren wir zu der Voraussetzung zurück, dass die Differentialgleichung $\lambda^{\text {ter }}$ Ordnung $P(y)=0$ mit einer $\mu^{\text {ter }}$ Ordnung $\boldsymbol{Q}(y)=\mathbf{0}$ alle Integrale gemeinsam hat oder dass

$$
\text { (7.) } \quad P(y)=R(Q(y))
$$

ist. Wenn $R^{\prime}(z)$ und $R(y, z)$ für $R(y)$ dieselbe Bedeutung haben, wie $P^{\prime}(z)$ und $P(y, z)$ für $P(y)$, und wenn $w$ irgend ein Integral der Differentialgleichung $\boldsymbol{R}^{\prime}(z)=0$ ist, so ist (4.)

$$
w \boldsymbol{R}(y)=\frac{d}{d x}(R(y, w)) .
$$

Ersetzt man in dieser Identität $y$ durch $Q(y)$, so erhält man

$$
w P(y)=\frac{d}{d x} R(Q(y), w) \text {. }
$$

Daraus geht hervor, dass $w$ ein Multiplicator von $P(y)=0$ ist, also der Differentialgleichung $P^{\prime}(z)=0$ genügt. Dieselbe hat also mit der Differentialgleichung $(\lambda-\mu)^{\text {ter }}$ Ordnung $R^{\prime}(z)=0$ alle Integrale gemeinsam, womit das oben ausgesprochene Theorem bewiesen ist. Beiläufig ergiebt sich noch der Satz:

II. Wenn die lineare Differentialgleichung $P(y)=0$ mit $Q(y)=0$ alle Integrale gemeinsam hat und $P(y)=R^{\prime}(Q(y))$ ist, so ist jeder Multiplicator von $\boldsymbol{R}(\boldsymbol{y})=\mathbf{0}$ auch ein Multiplicator von $\boldsymbol{P}(\boldsymbol{y})=0$.

Da die Differentialgleichung $\lambda_{\text {ter }}$ Ordnung $P^{\prime}(z)=0$ mit der $\nu^{\text {ter }}$ Ord- 
nung $R^{\prime}(z)=0$ alle Integrale gemeinsam hat, so ist $\boldsymbol{P}^{\prime}(z)=\boldsymbol{S}^{\prime}\left(\boldsymbol{R}^{\prime}(z)\right)$, wo $\boldsymbol{S}^{\prime}(\boldsymbol{z})$ ein Differentialausdruck $\mu^{\text {ter }}$ Ordnung ist. Ebenso aber, wie aus $P(y)=R(Q(y))$ folgt $P^{\prime}(z)=S^{\prime}\left(R^{\prime}(z)\right)$, ergiebt sich aus dieser Gleichung $P(y)=T(S(y))$. Daher steht zu vermuthen, dass $S(y)=Q(y)$ ist. Dies beweisen wir folgendermassen :

Die Identität (5.) lautet für die Differentialgleichung $R(y)=0$

$$
\frac{d}{d x} R(y, z)=z R(y)-(-1)^{v} y R^{\prime}(z) \text {. }
$$

Ersetzt man darin $y$ durch: $Q(y)$, so erhält man

$$
(-1)^{v} R^{\prime}(z) Q(y)=z P(y)-\frac{d}{d x} R(Q(y), z) \text {. }
$$

Nimmt man für $z$ irgend ein Integral $v$ der Differentialgleichung $P^{\prime}(z)=0$, so ergiebt sich daraus und aus Gleichung (4.)

$$
(-1)^{\nu} R^{\prime}(v) Q(y)=\frac{d}{d x}[P(y, v)-R(Q(y), v)] .
$$

Folglich ist $R^{\prime}(v)$ ein Multiplicator der Differentialgleichung $Q(y)=0$. Ist also $Q^{\prime}(z)=0$ die lineare Differentialgleichung $\mu^{\text {ter }}$ Ordnung, der die Multiplicatoren von $Q(y)=0$ genügen, so ist

$$
Q^{\prime}\left(R^{\prime}(v)\right)=0 \text {. }
$$

Mithin wird die lineare Differentialgleichung $\lambda^{\text {ter }}$ Ordnung

$$
Q^{\prime}\left(R^{\prime}(z)\right)=0
$$

in welcher der Coefficient von $z^{(\lambda)}$ gleich Eins ist, durch alle Integrale $v$ der Differentialgleichung $\lambda^{\text {ter }}$ Ordnung $P^{\prime}(z)=0$ befriedigt und muss daher mit derselben identisch sein.

Wir können folglich den Satz aussprechen:

III. Genügen die Multiplicatoren der linearen Differentialgleichungen

$$
P(y)=0, \quad Q(y)=0, \quad R(y)=0
$$

den linearen Differentialgleichungen

$$
P^{\prime}(y)=0, \quad Q^{\prime}(y)=0, \quad R^{\prime}(y)=0,
$$

so muss, wenn

ist, auch

$$
P(y)=R(Q(y))
$$

sein.

$$
P^{\prime}(\boldsymbol{y})=Q^{\prime}\left(\boldsymbol{R}^{\prime}(y)\right)
$$

Um einen besseren Einblick in das Wesen dieses Satzes zu gewähren, 
will ich noch einen anderen Beweis für denselben mittheilen, der mehr rechnend verfährt. Ist $\frac{1}{v_{0}}$ ein Integral der Differentialgleichung $P(y)=0$, in welcher der Coefficient von $y^{(\lambda)}$ nicht gleich Eins zu sein braucht, und setzt man

$$
y=\frac{1}{v_{0}} \int z d x,
$$

so ergiebt sich für $z$ eine lineare Differentialgleichung $(\lambda-1)^{\text {ter }}$ Ordnung $Q(z)=0$. Wird der Coefficient von $z^{(\lambda-1)}$ in $Q(z)$ gleich dem von $y^{(\lambda)}$ in $P(z)$, dividirt durch $v_{1}$, angenommen, so ist

$$
P(y)=Q\left(\frac{d v_{0} y}{d x}\right) \text {. }
$$

Indem man diese Umformung wiederholt anwendet, bringt man $P(y)$ auf die Form

$$
P(y)=v_{\lambda} \frac{d}{d x} v_{\lambda-1} \frac{d}{d x} v_{\lambda-2} \cdots \frac{d}{d x} v_{1} \frac{d}{d x} v_{0} y .
$$

Daher ist $\frac{1}{v_{\lambda}}$ ein Multiplicator der Differentialgleichung $P(y)=0$. Um aber ihre sämmtlichen Multiplicatoren zu ermitteln, ist es bei dieser Form von $P(y)$ am einfachsten, das Lagrangesche Verfahren der partiellen Integration anzuwenden. Es ist nämlich

$$
\begin{gathered}
\int z P(y) d x \\
=\left(v_{\lambda} z\right)\left(v_{\lambda-1} \frac{d}{d x} v_{\lambda-2} \cdots \frac{d}{d x} v_{0} y\right)-\int\left(v_{\lambda-1} \frac{d}{d x} v_{\lambda} z\right)\left(\frac{d}{d x} v_{\lambda-2} \frac{d}{d x} v_{\lambda-3} \cdots \frac{d}{d x} v_{0} y\right) d x .
\end{gathered}
$$

Setzt man daher zur Abkürzung

$$
\begin{gathered}
P(y, z)= \\
\left(v_{\lambda} z\right)\left(v_{\lambda-1} \frac{d}{d x} v_{\lambda-2} \cdots \frac{d}{d x} v_{0} y\right)-\left(v_{\lambda-1} \frac{d}{d x} v_{\lambda} z\right)\left(v_{\lambda-2} \frac{d}{d x} v_{\lambda-3} \cdots \frac{d}{d x} v_{0} y\right) \\
+\left(v_{\lambda-2} \frac{d}{d x} v_{\lambda-1} \frac{d}{d x} v_{\lambda} z\right)\left(v_{\lambda-3} \frac{d}{d x} v_{\lambda-4} \cdots \frac{d}{d x} v_{0} y\right)-\cdots+(-1)^{\lambda-1}\left(v_{1} \frac{d}{d x} v_{2} \cdots \frac{d}{d x} v_{\lambda} z\right)\left(v_{0} y\right)
\end{gathered}
$$

und

$$
P^{\prime}(z)=v_{0} \frac{d}{d x} v_{1} \frac{d}{d x} v_{2} \cdots \frac{d}{d x} v_{\lambda} z
$$

so gelangt man durch wiederholte Anwendung der partiellen Integration zu der Gleichung

$$
\int z P(y) d x=P(y, z)+(-1)^{2} \int y \dot{P}^{\prime}(z) d x,
$$


oder

$$
\frac{d}{d x} P(y, z)=z P(y)-(-1)^{\lambda} y P^{\prime}(z) .
$$

Daher ist $P^{\prime}(z)=0$ die Differentialgleichung, der die Multiplicatoren von $P(y)=0$ genügen müssen. Es gilt also der Satz:

IV. Die Multiplicatoren der linearen Differentialgleichung

$$
v_{\lambda} \frac{d}{d x} v_{\lambda-1} \frac{d}{d x} v_{\lambda-2} \cdots \frac{d}{d x} v_{1} \frac{d}{d x} v_{0} y=0
$$

genügen der linearen Differentialgleichung

$$
v_{1} \frac{d}{d x} v_{1} \frac{d}{d x} v_{2} \cdots \frac{d}{d x} v_{\lambda-1} \frac{d}{d x} v_{\lambda} y=0 .
$$

Setzt man nun

$$
\begin{aligned}
& Q(y)=v_{\mu} \frac{d}{d x} v_{\mu-1} \cdots \frac{d}{d x} v_{1} \frac{d}{d x} v_{1} y, \\
& R(y)=v_{\lambda} \frac{d}{d x} v_{\lambda-1} \cdots \frac{d}{d x} v_{\mu+1} \frac{d}{d x} y,
\end{aligned}
$$

so ist

$$
\boldsymbol{P}(\boldsymbol{y})=\boldsymbol{R}(\boldsymbol{Q}(\boldsymbol{y}))
$$

In Folge des eben hergeleiteten Satzes ist damn aber

$$
\begin{aligned}
& Q^{\prime}(z)=v_{0} \frac{d}{d x} v_{1} \quad \cdots \frac{d}{d x} v_{\mu-1} \frac{d}{d x} v_{\mu} z, \\
& R^{\prime}(z)=\frac{d}{d x} v_{\mu+1} \cdots \frac{d}{d x} v_{\lambda-1} \frac{d}{d x} v_{\lambda} z
\end{aligned}
$$

und daher

$$
P^{\prime}(\boldsymbol{z})=Q^{\prime}\left(R^{\prime}(z)\right) \text {. }
$$

Umgekehrt kann man auch durch wiederholte Anwendung dieses Satzes das Theorem (IV.) ableiten.

S. 8.

Wir wollen hier noch einen anderen Beweis für das erste Theorem des vorigen Paragraphen mittheilen, welcher dasselbe mit der in $\$ .4$ entwickelten Lösung unseres Problems in Zusammenhang bringt und über die Multiplicatoren einer linearen Differentialgleichung weitere Aufschlüsse giebt.

Sind $y_{1}, y_{2}, \ldots y_{\lambda}$ irgend $\lambda$ unter einander unabhängige Integrale der linearen Differentialgleichung $\lambda$ ter Ordnung $P(y)=0$, so sind die $\lambda$ Functionen Journal für Mathematik Bd. LXXVI. Heft 3. 
$z_{1}, z_{2}, \ldots z_{\lambda}$, welche durch die Gleichungen

$$
\begin{array}{rcc}
(1 .) & z_{1} y_{1}+z_{2} y_{2}+\cdots+z_{\lambda} y_{\lambda}=0, \\
(2 .) \quad z_{1} y_{1}^{(1)}+z_{2} y_{2}^{(1)}+\cdots+z_{\lambda} y_{\lambda}^{(1)}=0, & \cdot \cdots \\
(\lambda-1 .) & z_{1} y_{1}^{(\lambda-2)}+z_{2} y_{2}^{(\lambda-2)}+\cdots+z_{\lambda} y_{\lambda}^{(\lambda-2)}=0 \\
(\lambda .) & z_{1} y_{1}^{(\lambda-1)}+z_{2} y_{2}^{(\lambda-1)}+\cdots+z_{\lambda} y_{\lambda}^{(\lambda-1)}=1
\end{array}
$$

bestimmt sind, ebenfalls unter einander unabhängig. Denn bestände zwischen ihnen eine Relation von der Form

$$
\text { (0.) } z_{1} c_{1}+z_{2} c_{2}+\cdots+z_{\lambda} c_{\lambda}=0 \text {, }
$$

so würde sich aus den Gleichungen $(0),.(1),. \ldots(\lambda-1$.) ergeben, dass die Determinante

$$
\Sigma \pm c_{1} y_{2} y_{3}^{(1)} \ldots y_{\lambda-1}^{(\lambda-3)} y_{\lambda}^{(\lambda-2)}=0
$$

wäre. Daher müsste auch ihre Ableitung

$$
\Sigma \pm \dot{c}_{1} y_{2} y_{3}^{(1)} \ldots y_{\lambda-1}^{(\lambda-3)} y_{\lambda}^{(\lambda-1)}=0
$$

sein. Dann würden aber die Gleichungen (0.), (1.), ... ( $\lambda-2$.) zur Folge haben, dass auch der Ausdruck

$$
z_{1} y_{1}^{(\lambda-1)}+z_{2} y_{2}^{(\lambda-1)}+\cdots+z_{\lambda} y_{\lambda}^{(\lambda-1)}
$$

verschwände, während er doch den Werth 1 hat.

Die Functionen $y_{1}, \ldots y_{x-1}, y_{x+1}, \ldots y_{\lambda}$ genügen der linearen Differentialgleichung $(\lambda-1)^{\text {ter }}$ Ordnung

$$
\left(\Sigma \pm y^{\lambda-1} y_{1}^{\lambda-2} \ldots y_{x-1}^{\lambda-x} y_{x+1}^{\lambda-\lambda-1} \ldots y_{\lambda}\right):\left(\Sigma \pm y_{1}^{\lambda-2} \ldots y_{x-1}^{\lambda-x} y_{x+1}^{\lambda-x-1} \ldots y_{\lambda}\right)=0
$$

deren linke Seite wir mit

$$
Q(y)=y^{(\lambda-1)}+q_{1} y^{\lambda-2}+\cdots+q_{\lambda-1} y
$$

bezeichnen wollen. Wenn man die Gleichungen (1.), (2.), ... ( $\left.\lambda_{\text {. }}\right)$ der Reihe nach mit $q_{\lambda-1}, q_{\lambda-2}, \ldots q_{1}, 1$ multiplicirt und dann addirt, so erhält man

$$
z_{x} Q\left(y_{x}\right)=1, \quad z_{x}=\frac{1}{Q\left(y_{x}\right)} .
$$

Daher ist $z_{x}$ ein Multiplicator der Differentialgleichung $P(y)=0$. (Vergl. dì Ahh des Herrn Thomé, dieses Journal, Bd. 75, S. 271.) Denn die Differentialgleichung $\lambda^{\text {ter }}$ Ordnung

$$
\frac{d}{d x} \frac{Q(y)}{Q\left(y_{x}\right)}=0
$$

wird durch die $\lambda$ von einander unabhängigen Integrale $y_{1}, y_{2}, \ldots y_{2}$ der 
Differentialgleichung $P(y)=0$ befriedigt und mithin ist

$$
\frac{P(y)}{Q\left(y_{x}\right)}=\frac{d}{d x} \frac{Q(y)}{Q\left(y_{x}\right)} \text {. }
$$

Die Functionen $z_{1}, z_{2}, \ldots z_{\lambda}$ sind also $\lambda$ unter einander unabhängige Multiplicatoren der Differentialgleichung $P(y)=0$. Auch zu jedem andern System von $\lambda$ unter einander unabhängigen Integralen $\eta_{1}, \eta_{2}, \ldots \eta_{\lambda}$ dieser Differentialgleichung kann man mittelst der Gleichungen (1.), (2.), ...( $\left.\lambda_{\text {. }}\right) \lambda$ von einander unabhängige Multiplicatoren $\zeta_{1}, \zeta_{2}, \ldots \zeta_{\lambda}$ ermitteln. 'Ist nun

$$
\text { (A.) } y_{x}=a_{x 1} \eta_{1}+a_{x 2} \eta_{2}+\cdots+a_{x \lambda} \eta_{\lambda},
$$

so ergiebt sich aus den Gleichungen (1.), (2.), ...( $\left.\lambda_{\text {. }}\right)$

$$
\begin{gathered}
\Sigma\left(a_{1 x} z_{1}+a_{2 x} z_{2}+\cdots+a_{\lambda x} z_{\lambda}\right) \eta_{x}=0, \\
\Sigma\left(a_{1 x} z_{1}+a_{2 x} z_{2}+\cdots+a_{\lambda x} z_{\lambda}\right) \eta_{x}^{(1)}=0, \\
\cdot \cdot \cdot \cdot \cdot \cdot \cdot \\
\Sigma\left(a_{1 x} z_{1}+a_{2 x} z_{2}+\cdots+a_{\lambda x} z_{\lambda}\right) \eta_{x}^{(\lambda-2)}=0 \\
\Sigma\left(a_{1 x} z_{1}+a_{2 x} z_{2}+\cdots+a_{\lambda x} z_{\lambda}\right) \eta_{x}^{(\lambda-1)}=1 .
\end{gathered}
$$

Daher ist

$$
a_{1 x} z_{1}+a_{2 x} z_{2}+\cdots+a_{\lambda x} z_{\lambda}=\zeta_{x} \text {. }
$$

Bezeichnet man also in der Determinante $\Sigma \pm \omega_{11} a_{22} \ldots a_{2,2}$ den Coefficienten von $a_{\alpha \beta}$, dividirt durch die Determinante, mit $b_{\alpha \beta}$, so ist

(B.) $\quad z_{x}=b_{x 1} \zeta_{1}+b_{x^{2}} \zeta_{2}+\cdots+b_{x \lambda} \zeta_{\lambda}$.

Daraus schliesst man mit Hülfe des in $\$$. 2 bewiesenen Satzes, dass $z_{1}$, $z_{2}, \ldots z_{\lambda}$ die Integrale einer linearen Differentialgleichung $\lambda$ ter Ordnung $P^{\prime}(z)=0$ mit rationalen Coefficienten sind.

Wenn nun die Differentialgleichung $\lambda^{\text {ter }}$ Ordnung $P(y)=0$ mit einer $\mu^{\text {ter }}$ Ordnung von derselben Form $Q(y)=0$ alle Integrale gemeinsam hat, so kann man in jedes Fundamentalsystem von Integralen der ersten Differentialgleichung $\mu$ von einander unabhängige Integrale der zweiten aufnehmen. Sind aber $y_{1}, y_{2}, \ldots y_{\mu}$ und $\eta_{1}, \eta_{2}, \ldots \eta_{\mu}$ zwei Fundamentalsysteme von Integralen der Differentialgleichung $Q(y)=0$, so bestehen zwischen ihnen allein $\mu$ lineare Relationen, und daher muss $a_{\alpha \beta}=0$ sein, wenn $\alpha<\mu+1$ und $\beta>\mu$ ist. Mithin ist $b_{\alpha \beta}=0$, wenn $\alpha>\mu$ und $\beta<\mu+1$ ist. Denn eine Determinante $(\lambda-1)^{\text {ten }}$ Grades muss identisch verschwinden, wenn in ihr alle Elemente, welche $\mu$ Colonnen mit mehr als $\lambda-1-\mu$ Zeilen gemeinsam haben, gleich Null sind. Zwischen $z_{\mu+1}, z_{\mu+2}, \ldots z_{\lambda}$ und $\zeta_{\mu+1}, \zeta_{\mu+2}, \ldots \zeta_{\lambda}$ 
bestehen also $\lambda-\mu$ lineare Gleichungen. Da nun unter den sämmtlichen verschiedenen $Z$ weigen der Functionen $y_{1}, y_{2}, \ldots y_{\mu}$ nur $\mu$ von einander unabhăngige enthalten sind, so lassen sich alle Zweige der Functionen $z_{\mu+1}$, $z_{\mu+2}, \ldots z_{\lambda}$ durch diese $\lambda-\mu$ Zweige linear ausdrücken. Dieselben genügen daher einer linearen Differentialgleichung $(\lambda-\mu)^{\text {ter }}$ Ordnung, mit der die Differentialgleichung $P^{\prime}(z)=0$ alle Integrale gemeinsam hat.

\$. 9.

Bisher haben wir nur solche Differentialgleichungen betrachtet, deren Integrale nur eine endliche Anzahl singulärer Stellen haben, und an jeder derselben, $a$, mit einer endlichen Potenz von $x-a$ (oder $\frac{1}{x}$ für $a=\infty$ ) multiplicirt, endlich werden. Um den Satz, der hier noch bewiesen werden soll, nicht unnöthigen Beschränkungen unterwerfen zu müssen, wollen wir jetzt allgemein lineare Differentialgleichungen in Betracht ziehen, deren Coefficienten überall eindeutige Functionen sind, und eine solche irreductibel nennen, wenn sie mit keinér Differentialgleichung niedrigerer Ordnung, deren Coefficienten ebenfalls überall eindeutige Functionen sind, ein Integral gemeinsam hat. Die in den Paragraphen 2, 3 und 6 entwickelten Sätze lassen sich genau nach denselben Methoden auch für solche Differentialgleichungen beweisen.

Wenn eine Differentialgleichung $P=0$ von der früher vorausgesetzten Form in dem früheren Sinne reductibel ist, so ist sie es auch in dem jetzigen Sinne. Wenn sie ferner nach der neuen Definition reductibel ist, so giebt es eine Differentialgleichung niedrigerer Ordnung, mit der sie alle Integrale gemeinsam hat, und die folglich zu der besonderen Klasse von Differentialgleichungen gehört, von der wir bis jetzt gehandelt haben. Daher ist die Differentialgleichung $P=0$ auch in dem früheren Sinne reductibel. Daraus folgt, dass eine Differentialgleichung von der bisher betrachteten Form nach der jetzigen Definition reduetibel oder irreductibel ist, je nachdem sie es nach der früheren war.

In der schon oben erwähnten Abhandlung beweist Herr Brassinne den Satz, dass eine Differentialgleichung reductibel ist, wenn sie zwei Integrale $y_{0}$ und $y_{1}$ besitzt, zwischen denen die Beziehung $y_{1}=x y_{0}$ besteht. Dieser Satz ist nur ein specieller Fall folgendes allgemeinen Theorems:

Wenn von zwei verschiedenen Integralen einer homogenen linearen 
Differentialgleichung mit eindeutigen Coefficienten das eine ein homogener linearer Differentialausdruck mit eindeutigen Coefficienten von dem andern ist, so ist die Differentialgleichung reductibel.

Seien $q_{1}, q_{1}, \ldots q_{\mu}$ eindeutige Functionen von $x$ und sei

$$
\boldsymbol{Q}(\boldsymbol{y})=q_{0} y^{(\mu)}+q_{1} y^{(\mu-1)}+\cdots+q_{\mu} y
$$

ein homogener linearer Differentialausdruck $\mu^{\text {ter }}$ Ordnung von $y$. Seien ferner $y_{0}$ und $y_{1}$ zwei, unter einander unabhängige Integrale der linearen Differentialgleichung

$$
P(y)=p_{0} y^{(\lambda)}+p_{1} y^{(\lambda-1)}+\cdots+p_{\lambda} y=0,
$$

deren Coefficienten eindeutige Functionen von $x$ sind. Zwischen diesen bestehe die Beziehung

$$
y_{1}=Q\left(y_{10}\right)
$$

Wenn dann, wider die Behauptung des obigen Satzes, $P(y)=0$ irreductibel ist, so hat die lineare Differentialgleichung $P(Q(y))=0$ mit $P(y)=0$ alle Integrale gemeinsam, weil sie eins, $y_{1}$, mit ihr gemeinsam bat. Ist daher $y$ irgend ein Integral der Differentialgleichung $P(y)=0$, so ist auch $Q(y)$ ein solches. Mithin wird diese Differentialgleichung durch die Functionen

$$
y_{0}, \quad Q\left(y_{0}\right), \quad Q\left(Q\left(y_{0}\right)\right)=Q^{2}\left(y_{0}\right), \quad Q\left(Q^{2}\left(y_{0}\right)\right)=Q^{3}\left(y_{0}\right), \ldots
$$

befriedigt. Da sie aber nicht mehr als $\lambda$ unter einander unabhängige Integrale haben kann, so muss es in der Reihe dieser Integrale eins, $Q^{\nu}\left(y_{0}\right)$, geben, welches sich durch die vorhergehenden linear ausdrücken lässt, während diese noch unter einander unabhängig sind, oder es muss eine Gleichung von der Form

$$
a_{0} y_{0}+a_{1} Q\left(y_{10}\right)+\cdots+a_{\nu} Q^{\nu}\left(y_{0}\right)=0
$$

bestehen, in der $a_{v}$ von Null verschieden ist, während zwischen den Functionen

$$
y_{0}, \quad y_{1}=Q\left(y_{0}\right), \ldots y_{v-1}=Q^{\nu-1}\left(y_{0}\right)
$$

keine homogene lineare Relation mit constanten Coefficienten besteht. Die Zahl $\nu$ muss, weil $y_{1}$ der Annahme nach ein von $y_{0}$ verschiedenes Integral ist, grösser als Eins sein. Ist $\nu<\lambda$, so lassen sich $\lambda-\nu$ Integrale, $y_{\nu}$, $y_{\nu+1}, \ldots y_{\lambda-1}$ finden, welche zusammen mit $y_{v}, y_{1}, \ldots y_{\nu-1}$ ein vollständiges System unter einander unabhăngiger Integrale bilden. Wir setzen nun

und

$$
f(r)=a_{0}+a_{1} r+\cdots+a_{\nu} r^{\nu}
$$

$$
\frac{f(r)-f(s)}{r-s}=f_{1}(r)+f_{2}(r) s+\cdots+f_{v}(r) s^{v-1},
$$


also

Ist ferner

$$
\begin{aligned}
& f_{1}(r)=a_{1}+a_{2} r+\cdots+a_{\nu} r^{\nu-1}, \\
& f_{2}(r)=a_{2}+a_{3} r+\cdots+a_{\nu} r^{\nu-2}, \\
& \dot{f_{\nu}}(r)=a_{\nu} .
\end{aligned}
$$

so ist

Da aber

$$
\boldsymbol{R}(\boldsymbol{y})=f_{1}(\boldsymbol{r}) \boldsymbol{y}+f_{2}(\boldsymbol{r}) \boldsymbol{Q}(\boldsymbol{y})+\cdots+f_{\nu}(\boldsymbol{r}) Q^{\nu-1}(y),
$$

$$
R(Q(y))=f_{1}(r) Q(y)+f_{2}(r) Q^{2}(y)+\cdots+f_{v}(r) Q^{\nu}(y) .
$$

$$
f_{\nu}(r) Q^{\nu}\left(y_{0}\right)=a_{\nu} Q^{\nu}\left(y_{0}\right)=-a_{0} y_{0}-a_{1} Q\left(y_{0}\right)-\cdots-a_{\nu-1} Q^{\nu-1}\left(y_{0}\right)
$$

ist, so folgt aus der letzten Gleichung

$$
\begin{aligned}
R\left(Q\left(y_{0}\right)\right) & =-a_{0} y_{0}+\left(f_{1}(r)-a_{1}\right) Q\left(y_{0}\right)+\cdots+\left(f_{v-1}(r)-a_{v-1}\right) Q^{\nu-1}\left(y_{0}\right) \\
& =r\left(f_{1}(r) y_{0}+f_{2}(r) Q\left(y_{0}\right)+\cdots+f_{\nu}(r) Q^{\nu-1}\left(y_{0}\right)\right)-f(r) y_{0} .
\end{aligned}
$$

Ist daher $r$ eine Wurzel der Gleichung $f(r)=0$, so ist

$$
\boldsymbol{R}\left(\boldsymbol{Q}\left(y_{0}\right)\right)=\boldsymbol{r} \boldsymbol{R}\left(y_{0}\right) \text {. }
$$

Mithin muss jedes Integral $y$ der irreductibeln Differentialgleichung $P(y)=0$ der Differentialgleichung

genügen. Daher ist

$$
\boldsymbol{R}(\boldsymbol{Q}(\boldsymbol{y}))=\boldsymbol{r} \boldsymbol{R}(\boldsymbol{y})
$$

$$
R\left(y_{2}\right)=R\left(Q\left(Q\left(y_{0}\right)\right)\right)=r R\left(Q\left(y_{0}\right)\right)=r^{2} R(y)
$$

und allgemein, wenn $x<v$ ist

$$
\boldsymbol{R}\left(\boldsymbol{y}_{*}\right)=\boldsymbol{r}^{*} \boldsymbol{R}\left(\boldsymbol{y}_{0}\right) \text {. }
$$

Wenn nun die Variable $x$ von einem bestimmten Punkte aus irgend einen geschlossenen Weg durchläuft, so muss sich $y_{0}$ in einen Ausdruck von der Form

$$
c_{0} y_{0}+c_{1} y_{1}+\cdots+c_{\nu-1} y_{\nu-1}+c_{\nu} y_{\nu}+\cdots+c_{\lambda-1} y_{\lambda-1}
$$

verwandeln. Die Function $\boldsymbol{R}\left(y_{0}\right)$ geht auf diesem Wege in

$$
\left(c_{0}+c_{1} r+\cdots+c_{\nu-1} r^{\nu-1}\right) R\left(y_{0}\right)+c_{\nu} R\left(y_{\nu}\right)+\cdots+c_{\lambda-1} R\left(y_{\lambda-1}\right)
$$

über, und folglich lassen sich alle Zweige dieser Function durch

$$
\boldsymbol{R}\left(\boldsymbol{y}_{0}\right), \boldsymbol{R}\left(y_{\nu}\right), \ldots \boldsymbol{R}\left(\boldsymbol{y}_{\lambda-1}\right)
$$

linear mit constanten Coefficienten ausdrücken. Daher befriedigt $\boldsymbol{R}\left(\boldsymbol{y}_{0}\right)$ eine lineare Differentialgleichung höchstens $(\lambda-\nu+1)^{\text {ter }}$ Ordnung mit eindeutigen Coefficienten. Die Differentialgleichung $P(y)_{i}^{\prime \prime}=0$ hat demnach mit einer Differentialgleichung niedrigerer Ordnung ein Integral

$$
R\left(y_{0}\right)=f_{1}(r) y_{0}+f_{2}(r) y_{1}+\cdots+f_{v}(r) y_{0-1}
$$

gemeinsam, kann also nicht irreductibel sein.

Berlin, den 24. April 1873. 